\title{
On thermomechanics of multilayered
}

beams

Preprint of the article published in International Journal of Engineering Science

155, October 2020, 103364

Raffaele Barretta,

Marko Čanađija,

Francesco Marotti de Sciarra

(C) 2020. This manuscript version is made available under the CC-BYNC-ND 4.0 license http://creativecommons .org/licenses/by-nc-nd/4.0/ 


\title{
On thermomechanics of multilayered beams
}

\author{
Raffaele Barretta ${ }^{\mathrm{a}}$, Marko Čanađija ${ }^{\mathrm{b}, *}$, Francesco Marotti de Sciarra ${ }^{\mathrm{a}}$ \\ ${ }^{a}$ Department of Structures for Engineering and Architecture, University of Naples \\ Federico II, Via Claudio 21,80121 Naples, Italy \\ ${ }^{b}$ University of Rijeka, Faculty of Engineering, Department of Engineering Mechanics, \\ Vukovarska 58, 51000 Rijeka, Croatia
}

\begin{abstract}
In this paper, the mechanical behavior of multilayered small-scale beams in nonisothermal environment is investigated. Scale phenomena are modeled by means of the mathematically well-posed and experimentally consistent stress-driven integral formulation of elasticity. The present research extends the treatment in [1] confined to elastically homogeneous nano-scopic structures. It is shown that the non-locality leads to a complex coupling between axial and transverse elastic displacements. Such a size-dependent phenomenon makes the solution of the relevant nonlocal thermoelastostatic problem, governed by a system of two ordinary differential equations with ten standard boundary conditions and non-classical constitutive boundary conditions, significantly more involved with respect to treatments in literature. Thus, a novel solution methodology, based on Laplace transforms, is proposed and illustrated by examining simple structural schemes of current
\end{abstract}

\footnotetext{
${ }^{*}$ Corresponding author. Tel.: +385-51-651-496; Fax.: +385-51-651-490

Email address: marko.canadija@riteh.hr (Marko Čanađija )
} 
applicative interest in Nanomechanics and Nanotechnology.

Keywords: Multilayered beams, nonlocal thermoelasticity, Laplace

transforms, size effects, nanocomposites, MEMS/NEMS.

\section{Introduction, motivation and outline}

Analysis, modelling and assessment of elastic responses of small-scale structures has been a subject of great interest in the community of Engineering Science [2] due to the need to significantly design and optimize nanocomposites [3-7] and smaller and smaller technological devices [8 15]. So, to describe the deformation of advanced materials and new-generation structural systems, one needs to modify existing elasticity continuum methodologies, and non-local mechanics seems to be a promising choice to adequately capture technically important size effets [16-21] in comparison with computationally expensive atomistic strategies [22].

Like in the case of macroscopical structures, a combination of different materials in a nano- or micro-structure can lead toward superior mechanical behavior [23 25]. Composite beams can offer more possibilities than homogeneous structures [26 31]. This has a price - more complex models are needed to describe such problems which are even more harder to solve if nonisothermal environmental conditions are involved [1, 32 34].

Composite beams are a potential candidate for a wide variety of nanoscopic systems. In the present research, we set a focus on 1D continua made of several layers of different materials - multilayered beams. The literature mostly 
deals with a special class of such structures that are known as the sandwich beams [35]. A sandwich beam can be described by a central core layer, mostly thicker than two layers placed at the top and bottom surface. The thinner layers are often referred to as skins and the thickness is usually selected so the cross-section is symmetric.

Sandwich beams operate in many engineering applications. For instance, piezoelectric effects can be involved, so these are also included in the formulation [36 38]. A combination of sandwich and functionally graded (FG) materials including thermal effects are also considered and stability issues in the presence of dynamic effects are analyzed in [39, 40]. For the influence of thermal phenomena in wave propagation analysis in FG sandwich beams, see [41]. Nonlinear vibration in the case of sandwich beams that are graded with carbon nanotubes is researched in [42]. Free vibration of curved sandwichFG beams, again with various patterns of carbon nanotube distribution are examined in [43, piezoelectric and electrostatic actuation of laminated microarches in [44] and influence of a viscoelastic core in [45]. As a special case, formulations developed for vibrations of curved sandwich beams can be applied to straight beams by considering a very large curvature radius [46].

Apart from the gradient-based methods deriving from Eringen-type strategies [47] used in the aforementioned formulations, other methodologies are also resorted to. For instance, Joule heating in three-layered nanobeams was analyzed by modified couple stress theory in [48]. Mesh-free methods can be applied to nanocomposite sandwich plates under thermomechanical loads as 
well, see [49] for an illustration in the case of two-dimensional structures.

It is known that different properties of each particular layer lead towards the shift of neutral surface [50]. The effect exists in FG structures as well, see [51] for beams and initial contributions [52 54] for plates. Simplifications involving a symmetric structure of sandwich beam layers [37] conveniently avoid complications related to the introduction of the neutral surface shift, as it will be shown later. The same simplification benefits are obtained in FG beams graded only along axial direction [55]. Another approach is to simply disregard the shift in asymmetrical problems. For instance, see [56, 57] for research regarding nonlinear dynamics including temperature effects, dynamics influenced by imperfections in FG beams [31] and [58] for a comparison of Bernoulli-Euler and Timoshenko beam vibrations in three-layered beams of different thicknesses. Large differences in layers' thicknesses are also exploited for further simplifications [59] since some terms in the formulation can be disregraded as small.

The research at hand does not resort to such simplifications thus aiming at a more general and accurate solution. Two main novel aspects are involved. First of all, an existing isothermal model of non-local multilayered beams [60] is extended toward the nonisothermal regime. In contrast to gradient-based models described above, the present approach relies on the nonlocal stressdriven integral formulation developed in [16, 17, 61] and extended to FG nanobeams in [62, 63]. The formulation circumvents all known paradoxes and difficulties of non-local mechanics. Secondly, the presence of coupling 
between axial and transverse problems makes the solution of the underlying differential equations system much more complex and difficult to solve. As a non-standard approach to this issue in the absence of dynamical effects, Laplace transforms are exploited.

The outline of the paper is as follows. In the first part, preliminary considerations about geometric and material assumptions are given. BernoulliEuler kinematics for the nonisothermal regime follows, after which the nonlocal constitutive model is presented. Starting from the equilibrium conditions, the strict variational methodology is applied to formulate the governing differential equations accompanied by suitable sets of classical and constitutive boundary conditions. Solution procedures are described in the subsequent section. Examples section demonstrates the model behavior and provides a comprehensive analysis of the developed case-studies. Main findings and implications are summarized in the concluding section.

\section{Preliminaries}

\subsection{Geometry, materials and coordinate system}

This research considers multilayered beams, that is beams consisting of $n$ layers. The model will accommodate beams in which each layer is made of a different material and has a rectangular cross-section of different dimensions. The beam has length $L$ and it is assumed that no spatial changes take place regarding cross-section and material along the beam axis. Height and width

of each layer will be denoted by $h_{i}$ and $b_{i}, i \in\{1,2, \ldots, n\}$, respectively. Only 
cross-sections symmetric with respect to one axis will be considered. Each layer is assumed to be composed of elastic material with Young's modulus $E_{i}$ and coefficient of thermal expansion $\alpha_{i}$.

The coordinate system is defined in the following manner. The longitudinal axis is denoted by $x$, so the cross-section is positioned in the $y-z$ plane. Bending is assumed to take place in the $x-z$ plane. Consequently, the coordinates of upper and lower surfaces of a layer $i$ will be related to its height as $h_{i}=z_{i}-z_{i-1}$. The bottom of the first layer will be defined by the coordinate $z_{0}$.

\subsection{Nonisothermal multilayered beam kinematics}

One of the central problems in beams with stiffness varying along the bending direction $(z)$ is that the neutral surface does not necessarily include the cross-sectional centroid [51, 64, 65]. Therefore, the issue known as the neutral surface shift appears in the present case as well. The shift will be denoted by $\zeta_{0}$. Displacements along $y$ and $z$ directions are $v(x)$ and $w(x)$. Due to the plane bending assumption, $v(x)=0$. The remaining axial displacement field of a beam depends both on longitudinal and transverse coordinates. It is described by:

$$
u(x, z)=u_{0}(x)+\varphi(x)\left(z-\zeta_{0}\right)=\frac{1}{A} \int_{\Omega} u(x, z) \mathrm{d} A+\varphi(x)\left(z-\zeta_{0}\right),
$$

where the integral term represents the average axial displacement in the cross-section $x$. For a multilayered beam, the average axial displacement 
of a cross-section at $x$ is $u_{0}(x)=\frac{1}{A} \sum_{i=1}^{n} b_{i} \int_{z_{i-1}}^{z_{i}} u(x, z) \mathrm{d} z$. Note that the term involving rotation $\varphi(x)\left(z-\zeta_{0}\right)$ differs from the standard one used in homogenous beams $\varphi(x) z$ by the neutral shift $\zeta_{0}$.

Invoking the Bernoulli-Euler hypothesis relates the first derivative of the transverse displacement $w(x)$ to the angle of cross-sectional rotation $\varphi(x)$ as:

$$
w^{(1)}(x)=-\varphi(x)
$$

This result can be used to express strains in a layer $i, z \in\left[z_{i-1}, z_{i}\right]$ as

$$
\varepsilon_{i}(x, z)=u_{0}^{(1)}-w^{(2)}\left(z-\zeta_{0}\right)=\varepsilon_{0}-w^{(2)}\left(z-\zeta_{0}\right), \quad \gamma_{x z}(x, z)=0 .
$$

Above, the apex ${ }^{(n)}$ denotes $n$-th derivative with respect to the longitudinal coordinate $x$.

The preceding formulation is generally valid in both isothermal and nonisothermal settings. Since the focus of the present model is on non-homogenous temperature fields $\Delta \theta(x, z)$, thermal effects should be introduced. To this end, additive decomposition of normal strain into mechanical part $\varepsilon_{i, \mathrm{M}}$ and thermal part $\varepsilon_{i, \mathrm{~T}}=\alpha_{i} \Delta \theta$ is introduced [32]:

$$
\varepsilon_{i}(x, z)=\varepsilon_{i, \mathrm{~T}}+\varepsilon_{i, \mathrm{M}} .
$$


Now, equalizing Eqs. (3, 4):

$$
\alpha_{i} \Delta \theta+\varepsilon_{i, \mathrm{M}}=\varepsilon_{0}-w^{(2)}\left(z-\zeta_{0}\right)
$$

where the mechanical strain $\varepsilon_{i, \mathrm{M}}$ is obtained from a specific non-local constitutive model.

\subsection{Non-local constitutive behavior}

The constitutive model for the mechanical strain $\varepsilon_{i, \mathrm{M}}$ is now provided. It was recently shown [64] that the thermodynamic framework suitable for the non-local beam behavior relies on the Gibbs potential. For a layer $i$, this potential has the form:

$$
\rho_{i} g_{i}\left(\sigma_{i}, \Delta \theta\right)=-\frac{1}{2} \sigma_{i} \int_{0}^{L} \phi_{\lambda, i}(x-\xi) E_{i}^{-1} \sigma_{i}(\xi, z) \mathrm{d} \xi-\sigma_{i} \alpha_{i} \Delta \theta
$$

where the kernel function $\phi_{\lambda, i}(x)$ is

$$
\phi_{\lambda, i}(x)=\frac{1}{2 L_{\lambda, i}} \exp \left(-\frac{|x|}{L_{\lambda, i}}\right)
$$

$\sigma_{i}=\sigma_{i}(x, z)$ is the normal stress and $L_{\lambda}=\lambda L$ is the characteristic length. Obviously, the characteristic length is defined in bounded domains of length $L$ multiplied by the non-dimensional small-size parameter $\lambda$. An overview of values of $\lambda$ for carbon nanotubes is given in [22]. Constitutive behavior for 
the normal strain is then obtained from:

$$
\varepsilon_{i}=-\partial_{\sigma_{i}} g_{i}
$$

Using Eq. (6), differentiation gives:

$$
\varepsilon_{i}(x, z)=\int_{0}^{L} \phi_{\lambda, i}(x-\xi) E_{i}^{-1} \sigma_{i}(\xi, z) \mathrm{d} \xi+\alpha_{i} \Delta \theta
$$

The first term on the right-hand side represents the mechanical part of the normal strain $\varepsilon_{i, \mathrm{M}}$, while the second one is thermal strain $\varepsilon_{i, \mathrm{~T}}$. This specific one-dimensional non-local stress-driven integral model was introduced in [16, 17. 61 for homogeneous temperature fields context and extended to nonhomogenous temperature fields in [1].

Evaluation of the latter integral is not straightforward [1, 66]. The solution

$$
\sigma_{i}=E\left(-L_{\lambda, i}^{2}\left(\varepsilon_{i}^{(2)}-\left(\alpha_{i} \Delta \theta\right)^{(2)}\right)+\varepsilon_{i}-\alpha_{i} \Delta \theta\right)
$$

has to accommodate the constraints at the beams' ends:

$$
\begin{aligned}
& E_{i} L_{\lambda, i}\left(\varepsilon_{i}^{(1)}-\left(\alpha_{i} \Delta \theta\right)^{(1)}\right)-E_{i}\left(\varepsilon_{i}-\alpha_{i} \Delta \theta\right)=\left.0\right|_{\text {at }(0, z)}, \\
& E_{i} L_{\lambda, i}\left(\varepsilon_{i}^{(1)}-\left(\alpha_{i} \Delta \theta\right)^{(1)}\right)+E_{i}\left(\varepsilon_{i}-\alpha_{i} \Delta \theta\right)=\left.0\right|_{\text {at }(L, z)} .
\end{aligned}
$$

These constraints are known as the constitutive boundary conditions (CBC) and are typically overlooked in gradient based formulations. However, as shown in [16, 17, 61], inclusion of CBC effectively solves well-known para- 
doxes observed in literature [67, 68].

\section{Equilibrium and constitutive boundary conditions}

With the foundations of the problem provided in the previous section, the link between displacements and stress resultants can be set-up. The formulation relies on linking displacements $u_{0}(x)$ and $w(x)$ to stress resultants $N(x)$ and $M(x)$, i.e. the axial force and the bending moment, respectively. With Eqs. (10, 11) being the starting point, the normal strain in replaced with displacements by enforcing Bernoulli-Euler kinematics, Eq. (3):

$$
\frac{\sigma}{E_{i}}=-L_{\lambda, i}^{2}\left(u_{0}^{(3)}-w^{(4)}\left(z-\zeta_{0}\right)-\alpha_{i} \Delta \theta\right)+u_{0}^{(1)}-w^{(2)}\left(z-\zeta_{0}\right)-\alpha_{i} \Delta \theta
$$

and constraints:

$$
\begin{aligned}
& E_{i} L_{\lambda, i}\left(u_{0}^{(2)}-w^{(3)}\left(z-\zeta_{0}\right)-\alpha_{i} \Delta \theta\right)-E_{i}\left(u_{0}^{(1)}-w^{(2)}\left(z-\zeta_{0}\right)-\alpha_{i} \Delta \theta\right)=\left.0\right|_{x=0}, \\
& E_{i} L_{\lambda, i}\left(u_{0}^{(2)}-w^{(3)}\left(z-\zeta_{0}\right)-\alpha_{i} \Delta \theta\right)+E_{i}\left(u_{0}^{(1)}-w^{(2)}\left(z-\zeta_{0}\right)-\alpha_{i} \Delta \theta\right)=\left.0\right|_{x=L} .
\end{aligned}
$$

In the second step, latter equations should be rearranged by means of standard equilibrium equations. For multilayered beams these equations provide stress resultants - the axial force and bending moment as:

$$
\begin{aligned}
& N(x)=\sum_{i=1}^{n} b_{i} \int_{z_{i-1}}^{z_{i}} \sigma(x, z) \mathrm{d} z \\
& M(x)=\sum_{i=1}^{n} b_{i} \int_{z_{i-1}}^{z_{i}} \sigma(x, z)\left(z-\zeta_{0}\right) \mathrm{d} z .
\end{aligned}
$$

Introducing stresses as defined by Eq. (12) and with notation defined in Boxes 
1., 2 and 3 it follows:

$$
N(x)=\mathbf{N} \cdot \mathbf{1}=\mathbf{N}_{\mathrm{M}} \cdot \mathbf{1}+\mathbf{N}_{\mathrm{T}} \cdot \mathbf{1},
$$

where

$$
\begin{aligned}
\mathbf{N}_{\mathrm{M}} \cdot \mathbf{1}= & -L^{2}\left(\mathbf{A} \odot \mathbf{E} \odot \boldsymbol{\lambda}^{2}\right) \cdot \mathbf{1} u_{0}^{(3)}+L^{2}\left(\mathbf{S} \odot \mathbf{E} \odot \boldsymbol{\lambda}^{\odot 2}\right) \cdot \mathbf{1} w^{(4)} \\
& +(\mathbf{E} \odot \mathbf{A}) \cdot \mathbf{1} u_{0}^{(1)}-(\mathbf{E} \odot \mathbf{S}) \cdot \mathbf{1} w^{(2)} \\
\mathbf{N}_{\mathrm{T}} \cdot \mathbf{1}= & \mathbf{b} \odot \mathbf{E} \odot \boldsymbol{\alpha} \odot \boldsymbol{\Theta}_{\mathrm{N}}
\end{aligned}
$$

and $\odot$ denotes the Hadamard product. If the notation in Box 3 is used, a more compact form is obtained:

$$
N(x)=-k_{E A}^{2 N L} u_{0}^{(3)}+k_{E S}^{2 N L} w^{(4)}+k_{E A} u_{0}^{(1)}-k_{E S} w^{(2)}+k_{T N} .
$$

The same procedure can be repeated for the equilibrium equation regarding the bending moment Eq. $(14)_{2}$.

$$
M(x)=\mathbf{M} \cdot \mathbf{1}=\mathbf{M}_{\mathrm{M}} \cdot \mathbf{1}+\mathbf{M}_{\mathrm{T}} \cdot \mathbf{1},
$$

where

$$
\begin{aligned}
\mathbf{M}_{\mathrm{M}} \cdot \mathbf{1}= & -L^{2}\left(\mathbf{S} \odot \mathbf{E} \odot \boldsymbol{\lambda}^{\odot 2}\right) \cdot \mathbf{1} u_{0}^{(3)}+L^{2}\left(\mathbf{I} \odot \mathbf{E} \odot \boldsymbol{\lambda}^{\odot 2}\right) \cdot \mathbf{1} w^{(4)} \\
& +(\mathbf{E} \odot \mathbf{S}) \cdot \mathbf{1} u_{0}^{(1)}-(\mathbf{E} \odot \mathbf{I}) \cdot \mathbf{1} w^{(2)} \\
\mathbf{M}_{\mathrm{T}} \cdot \mathbf{1}= & \mathbf{b} \odot \mathbf{E} \odot \boldsymbol{\alpha} \odot \boldsymbol{\Theta}_{\mathrm{M}}
\end{aligned}
$$


or

$$
M(x)=-k_{E S}^{2 N L} u_{0}^{(3)}+k_{E I}^{2 N L} w^{(4)}+k_{E S} u_{0}^{(1)}-k_{E I} w^{(2)}+k_{T M} .
$$

A similar procedure must be applied to the constitutive boundary conditions Eq. (13) which become for equilibrium of axial forces:

$$
\begin{aligned}
& L(\boldsymbol{\lambda} \odot \mathbf{E}) \cdot\left(u_{0}^{(2)} \mathbf{A}-w^{(3)} \mathbf{S}-\left(\boldsymbol{\alpha} \odot \boldsymbol{\Theta}_{\mathrm{N}}\right)^{(1)}\right)-\mathbf{E} \cdot\left(u_{0}^{(1)} \mathbf{A}-w^{(2)} \mathbf{S}-\boldsymbol{\alpha} \odot \boldsymbol{\Theta}_{\mathrm{N}}\right)=\left.0\right|_{x=0}, \\
& L(\boldsymbol{\lambda} \odot \mathbf{E}) \cdot\left(u_{0}^{(2)} \mathbf{A}-w^{(3)} \mathbf{S}-\left(\boldsymbol{\alpha} \odot \boldsymbol{\Theta}_{\mathrm{N}}\right)^{(1)}\right)+\mathbf{E} \cdot\left(u_{0}^{(1)} \mathbf{A}-w^{(2)} \mathbf{S}-\boldsymbol{\alpha} \odot \boldsymbol{\Theta}_{\mathrm{N}}\right)=\left.0\right|_{x=L}
\end{aligned}
$$

Likewise, for equilibrium of bending moments, the constitutive boundary conditions are:

$$
\begin{aligned}
& L(\boldsymbol{\lambda} \odot \mathbf{E}) \cdot\left(u_{0}^{(2)} \mathbf{S}-w^{(3)} \mathbf{I}-\left(\boldsymbol{\alpha} \odot \boldsymbol{\Theta}_{\mathrm{M}}\right)^{(1)}\right)-\mathbf{E} \cdot\left(u_{0}^{(1)} \mathbf{S}-w^{(2)} \mathbf{I}-\boldsymbol{\alpha} \odot \boldsymbol{\Theta}_{\mathrm{M}}\right)=\left.0\right|_{x=0}, \\
& L(\boldsymbol{\lambda} \odot \mathbf{E}) \cdot\left(u_{0}^{(2)} \mathbf{S}-w^{(3)} \mathbf{I}-\left(\boldsymbol{\alpha} \odot \boldsymbol{\Theta}_{\mathrm{M}}\right)^{(1)}\right)+\mathbf{E} \cdot\left(u_{0}^{(1)} \mathbf{S}-w^{(2)} \mathbf{I}-\boldsymbol{\alpha} \odot \boldsymbol{\Theta}_{\mathrm{M}}\right)=\left.0\right|_{x=L}
\end{aligned}
$$

With the notation in Box 3 two sets of constitutive boundary conditions are:

$$
\begin{aligned}
& \left(k_{E A}^{N L} u_{0}^{(2)}-k_{E S}^{N L} u_{0}^{(2)} w^{(3)}-k_{T N}^{N L}\right)-\left(k_{E A} u_{0}^{(1)}-k_{2 T N}\right)=\left.0\right|_{x=0} \\
& \left(k_{E A}^{N L} u_{0}^{(2)}-k_{E S}^{N L} u_{0}^{(2)} w^{(3)}-k_{T N}^{N L}\right)+\left(k_{E A} u_{0}^{(1)}-k_{2 T N}\right)=\left.0\right|_{x=L} \\
& \left(k_{E S}^{N L} u_{0}^{(2)}-k_{E I}^{N L} w^{(3)}-k_{T M}^{N L}\right)-\left(-k_{E I} w^{(2)}-k_{2 T M}\right)=\left.0\right|_{x=0} \\
& \left(k_{E S}^{N L} u_{0}^{(2)}-k_{E I}^{N L} w^{(3)}-k_{T M}^{N L}\right)+\left(-k_{E I} w^{(2)}-k_{2 T M}\right)=\left.0\right|_{x=L}
\end{aligned}
$$

This completes non-local stress-driven beam relations between displacements and stress resultants. 


\section{Box 1: Notation for geometry and material property vectors,}

$(\bullet) \in \mathbb{R}^{n}, i \in\{1,2, \ldots n\}$.

Geometry:

Widths of layers $\mathbf{b}$ :

$b_{i}$

Heights of layers $\mathbf{h}$ :

$h_{i}$

Cross-section areas of layers $\mathbf{A}=\mathbf{b} \odot \mathbf{h}: \quad A_{i}=b_{i} h_{i}$

First moments of area of layers $\mathbf{S}: \quad S_{i}=b_{i} \int_{z_{i-1}}^{z_{i}}\left(z-\zeta_{0}\right) \mathrm{d} z$

Second moments of area of layers $\mathbf{I}: \quad I_{i}=b_{i} \int_{z_{i-1}}^{z_{i}}\left(z-\zeta_{0}\right)^{2} \mathrm{~d} z$

Material:

Youngs' moduli E: $\quad E_{i}$

Coefficients of thermal expansion $\boldsymbol{\alpha}$ : $\quad \alpha_{i}$

Small-size parameters $\boldsymbol{\lambda}: \quad \lambda_{i}$ 
Box 2: Loading vectors, $(\bullet) \in \mathbb{R}^{n}, i \in\{1,2, \ldots n\}$

Temperature integral, axial part $\Theta_{\mathrm{N}}: \Theta_{\mathrm{N} i}=\int_{z_{i-1}}^{z_{i}} \Delta \theta(x, z) \mathrm{d} z$

Temperature integral, bending part $\Theta_{\mathrm{M}}: \Theta_{\mathrm{M} i}=\int_{z_{i-1}}^{z_{i}} \Delta \theta(x, z)\left(z-\zeta_{0}\right) \mathrm{d} z$ Axial force, mechanical part $\mathbf{N}_{\mathrm{M}}$ :

$$
\begin{aligned}
N_{\mathrm{M} i} & =b_{i} E_{i}\left(-L_{\lambda, i}^{2}\left(u_{0}^{(3)} h_{i}-w^{(4)} \int_{z_{i-1}}^{z_{i}}\left(z-\zeta_{0}\right) \mathrm{d} z\right)\right. \\
& \left.+u_{0}^{(1)} h_{i}-w^{(2)} \int_{z_{i-1}}^{z_{i}}\left(z-\zeta_{0}\right) \mathrm{d} z\right)
\end{aligned}
$$

Axial force, thermal part $\mathbf{N}_{\mathrm{T}}$ :

$N_{\mathrm{T} i}=b_{i} E_{i} \alpha_{i} \int_{z_{i-1}}^{z_{i}} \Delta \theta(x, z) \mathrm{d} z=b_{i} E_{i} \alpha_{i} \Theta_{\mathrm{N} i}$

Bending moment, mechanical part $\mathbf{M}_{\mathrm{M}}$ :

$$
\begin{aligned}
M_{\mathrm{M} i} & =b_{i} E_{i}\left(-L_{\lambda, i}^{2}\left(u_{0}^{(3)} h_{i}-w^{(4)} \int_{z_{i-1}}^{z_{i}}\left(z-\zeta_{0}\right) \mathrm{d} z\right)\right. \\
& \left.+u_{0}^{(1)} h_{i}-w^{(2)} \int_{z_{i-1}}^{z_{i}}\left(z-\zeta_{0}\right) \mathrm{d} z\right)
\end{aligned}
$$

Bending moment, thermal part $\mathbf{M}_{\mathrm{T}}$ :

$$
M_{\mathrm{T} i}=b_{i} E_{i} \alpha_{i} \int_{z_{i-1}}^{z_{i}} \Delta \theta(x, z)\left(z-\zeta_{0}\right) \mathrm{d} z=b_{i} E_{i} \alpha_{i} \Theta_{\mathrm{M} i}
$$




\section{Box 3: Notation for stiffnesses}

$$
\begin{aligned}
& k_{E A}=\sum_{i=1}^{n} b_{i} E_{i} \int_{z_{i-1}}^{z_{i}} \mathrm{~d} z=\sum_{i=1}^{n} A_{i} E_{i}=(\mathbf{E} \odot \mathbf{A}) \cdot \mathbf{1}=\mathbf{E} \cdot \mathbf{A}, \\
& k_{E S}=\sum_{i=1}^{n} b_{i} E_{i} \int_{z_{i-1}}^{z_{i}}\left(z-\zeta_{0}\right) \mathrm{d} z=(\mathbf{E} \odot \mathbf{S}) \cdot \mathbf{1}=\mathbf{E} \cdot \mathbf{S}, \\
& k_{E I}=\sum_{i=1}^{n} b_{i} E_{i} \int_{z_{i-1}}^{z_{i}}\left(z-\zeta_{0}\right)^{2} \mathrm{~d} z=(\mathbf{E} \odot \mathbf{I}) \cdot \mathbf{1}=\mathbf{E} \cdot \mathbf{I}, \\
& k_{E A}^{N L}=\sum_{i=1}^{n} b_{i} E_{i} h_{i} L_{\lambda, i}=L(\boldsymbol{\lambda} \odot \mathbf{E}) \cdot \mathbf{A} \\
& k_{E S}^{N L}=\sum_{i=1}^{n} b_{i} E_{i} L_{\lambda, i} \int_{z_{i-1}}^{z_{i}}\left(z-\zeta_{0}\right) \mathrm{d} z=L(\boldsymbol{\lambda} \odot \mathbf{E}) \cdot \mathbf{S} \\
& k_{E I}^{N L}=\sum_{i=1}^{n} b_{i} E_{i} L_{\lambda, i} \int_{z_{i-1}}^{z_{i}}\left(z-\zeta_{0}\right)^{2} \mathrm{~d} z=L(\boldsymbol{\lambda} \odot \mathbf{E}) \cdot \mathbf{I} \\
& k_{E A}^{2 N L}=\sum_{i=1}^{n} b_{i} E_{i} h_{i} L_{\lambda, i}^{2}=L^{2}\left(\mathbf{A} \odot \mathbf{E} \odot \boldsymbol{\lambda}^{\odot 2}\right) \cdot \mathbf{1}, \\
& k_{E S}^{2 N L}=\sum_{i=1}^{n} b_{i} E_{i} L_{\lambda, i}^{2} \int_{z_{i-1}}^{z_{i}}\left(z-\zeta_{0}\right) \mathrm{d} z=L^{2}(\mathbf{S} \odot \mathbf{E} \odot \boldsymbol{\lambda} \odot 2) \cdot \mathbf{1}, \\
& k_{E I}^{2 N L}=\sum_{i=1}^{n} b_{i} E_{i} L_{\lambda, i}^{2} \int_{z_{i-1}}^{z_{i}}\left(z-\zeta_{0}\right)^{2} \mathrm{~d} z=L^{2}(\mathbf{I} \odot \mathbf{E} \odot \boldsymbol{\lambda} \odot 2) \cdot \mathbf{1}, \\
& k_{T N}=\sum_{i=1}^{n} b_{i} E_{i} \alpha_{i} \int_{z_{i-1}}^{z_{i}} \Delta \theta(x, z) \mathrm{d} z=(\mathbf{b} \odot \mathbf{E}) \cdot\left(\boldsymbol{\alpha} \odot \boldsymbol{\Theta}_{\mathrm{N}}\right) \\
& k_{T M}=\sum_{i=1}^{n} b_{i} E_{i} \alpha_{i} \int_{z_{i-1}}^{z_{i}} \Delta \theta(x, z)\left(z-\zeta_{0}\right) \mathrm{d} z=(\mathbf{b} \odot \mathbf{E}) \cdot\left(\boldsymbol{\alpha} \odot \boldsymbol{\Theta}_{\mathrm{M}}\right) \\
& k_{T N}^{N L}=L(\boldsymbol{\lambda} \odot \mathbf{E}) \cdot\left(\boldsymbol{\alpha} \odot \boldsymbol{\Theta}_{\mathrm{N}}\right)^{(1)} \\
& k_{T M}^{N L}=L(\boldsymbol{\lambda} \odot \mathbf{E}) \cdot\left(\boldsymbol{\alpha} \odot \boldsymbol{\Theta}_{\mathrm{M}}\right)^{(1)} \\
& k_{2 T N}=\mathbf{E} \cdot\left(\boldsymbol{\alpha} \odot \boldsymbol{\Theta}_{\mathrm{N}}\right) \\
& k_{2 T M}=\mathbf{E} \cdot\left(\boldsymbol{\alpha} \odot \boldsymbol{\Theta}_{\mathrm{M}}\right)
\end{aligned}
$$

where:

vector of ones: $\mathbf{1}=\left\{\begin{array}{llll}1 & 1 & \ldots & 1\end{array}\right\}^{T}$ of size $n$

$\boldsymbol{\lambda}^{\odot 2}=\boldsymbol{\lambda} \odot \boldsymbol{\lambda}$

Symbol $\odot$ represents the Hadamard product of two vectors.

Symbol - denotes the scalar product of two vectors as usual. 


\section{Variational formulation}

The complete differential formulation augmented with boundary conditions is now obtained by enforcing the strict varational approach. The governing potential is defined by:

$$
\Pi\left(u_{0}, w\right)=U_{i}-U_{e}
$$

where the internal potential $U_{i}$ for a beam $\mathcal{B}$ is:

$$
U_{i}=\int_{\mathcal{B}} \int_{0}^{\varepsilon} \sigma \mathrm{d} \bar{\varepsilon} \mathrm{d} V
$$

The external potential $U_{e}$ is:

$$
\begin{aligned}
U_{e}= & \int_{L} q_{x} u_{0} \mathrm{~d} x+\int_{L} q_{z} w \mathrm{~d} x+\mathcal{N}_{0} u_{0}(0)+\mathcal{N}_{L} u_{0}(L) \\
& +\mathcal{T}_{0} w(0)+\mathcal{T}_{L} w(L)-\mathcal{M}_{0} w^{(1)}(0)-\mathcal{M}_{L} w^{(1)}(L)
\end{aligned}
$$

where $\mathcal{N}_{0}, \mathcal{N}_{L}$ and $\mathcal{T}_{0}, \mathcal{T}_{L}$ are external axial and transverse forces at $x \in$ $\{0, L\}$, respectively. $\mathcal{M}_{0}, \mathcal{M}_{L}$ are external moments at same positions. $q_{z}(x)$ and $q_{x}(x)$ are distributed transverse and axial loadings, respectively,

The internal potential should be transformed into a more suitable form involving stress resultants Eq. (14). To this end, Eq. (3) relating strains and displacements is introduced into the internal potential Eq. 25):

$$
U_{i}=\int_{L} N u_{0}^{(1)} \mathrm{d} x-\int_{L} M w^{(2)}\left(z-\zeta_{0}\right) \mathrm{d} x .
$$


Unknown displacement fields are obtained by invoking the stationarity of the minimum potential energy:

$$
\left(u_{0}, w\right)=\arg \inf _{u_{0}, w} \Pi\left(u_{0}, w\right)
$$

Application of the first stationarity condition $\delta_{u_{0}} \Pi=0$ gives:

$$
\delta_{u_{0}} \Pi=\int_{L} N \delta u_{0}^{(1)} \mathrm{d} x-\int_{L} q_{x} \delta u_{0} \mathrm{~d} x-\mathcal{N}_{0} \delta u_{0}(0)-\mathcal{N}_{L} \delta u_{0}(L)=0 .
$$

Performing integration by parts and grouping terms with virtual displacement $\delta u_{0}$ provides:

$$
N^{(1)}+q_{x}=0
$$

what upon application of Eq. (17) provides the governing differential equation:

$$
-k_{E A}^{2 N L} u_{0}^{(4)}+k_{E S}^{2 N L} w^{(5)}+k_{E A} u_{0}^{(2)}-k_{E S} w^{(3)}+k_{T N}^{(1)}+q_{x}=0 .
$$

Boundary conditions follow from considering terms with virtual displacements at the beam's end $\delta u_{0}(0)$ and $\delta u_{0}(L)$, again by virtue of Eq. (17):

$$
\begin{array}{ll}
\left.\left(-k_{E A}^{2 N L} u_{0}^{(3)}+k_{E S}^{2 N L} w^{(4)}+k_{E A} u_{0}^{(1)}-k_{E S} w^{(2)}+k_{T N}\right)\right|_{x=0}=-\mathcal{N}_{0} & \text { or prescribe } u_{0}(0), \\
\left.\left(-k_{E A}^{2 N L} u_{0}^{(3)}+k_{E S}^{2 N L} w^{(4)}+k_{E A} u_{0}^{(1)}-k_{E S} w^{(2)}+k_{T N}\right)\right|_{x=L}=\mathcal{N}_{L} & \text { or prescribe } u_{0}(L) .
\end{array}
$$

In addition to above boundary conditions, the constitutive boundary condi- 
tions Eqs. 21 must be also enforced.

Transverse displacements follow from the second stationary condition $\delta_{w} \Pi=0:$

$$
\delta_{w} \Pi=-\int_{L} M \delta w^{(2)} \mathrm{d} x-\int_{L} q_{z} \delta w \mathrm{~d} x-\mathcal{T}_{0} \delta w(0)-\mathcal{T}_{L} \delta w(L)+\mathcal{M}_{0} \delta w^{(1)}(0)+\mathcal{M}_{L} \delta w^{(1)}(L)=0 .
$$

Integrating by parts twice, with Eq. 201, and assembling terms with the virtual displacement $\delta w$ provides:

$$
-M^{(2)}-q_{z}=0
$$

or with Eq. (20):

$$
-k_{E S}^{2 N L} u_{0}^{(5)}+k_{E I}^{2 N L} w^{(6)}+k_{E S} u_{0}^{(3)}-k_{E I} w^{(4)}+k_{T M}^{(2)}+q_{z}=0 .
$$

Boundary conditions follow from terms with $\delta w$ at $x \in\{0, L\}$ and $\delta w^{(1)}$ at $x \in\{0, L\}:$

$$
\begin{array}{lr}
\left.\left(-k_{E S}^{2 N L} u_{0}^{(3)}+k_{E I}^{2 N L} w^{(4)}+k_{E S} u_{0}^{(1)}-k_{E I} w^{(2)}+k_{T M}\right)\right|_{x=0}=-\mathcal{M}_{0} & \text { or prescribe } w^{(1)}(0), \\
\left.\left(-k_{E S}^{2 N L} u_{0}^{(3)}+k_{E I}^{2 N L} w^{(4)}+k_{E S} u_{0}^{(1)}-k_{E I} w^{(2)}+k_{T M}\right)\right|_{x=L}=\mathcal{M}_{L} & \text { or prescribe } w^{(1)}(L)
\end{array}
$$


while the corresponding part for $\delta w$ at $x \in\{0, L\}$ gives:

$$
\begin{array}{lr}
\left.\left(-k_{E S}^{2 N L} u_{0}^{(4)}+k_{E I}^{2 N L} w^{(5)}+k_{E S} u_{0}^{(2)}-k_{E I} w^{(3)}+k_{T M}^{(1)}\right)\right|_{x=0}=-\mathcal{T}_{0} & \text { or prescribe } w(0) \\
\left.\left(-k_{E S}^{2 N L} u_{0}^{(4)}+k_{E I}^{2 N L} w^{(5)}+k_{E S} u_{0}^{(2)}-k_{E I} w^{(3)}+k_{T M}^{(1)}\right)\right|_{x=L}=\mathcal{T}_{L} & \text { or prescribe } w(L) .
\end{array}
$$

Similarly to the axial displacements case, the constitutive boundary conditions Eqs. 22 must be respected as well. Finally, the axial force and bending moment can be obtained after solving for displacements by means Eq. 17 , 20 .

\subsection{Position of the neutral surface and coupling}

To determine the position of the neutral surface $\zeta_{0}$ we start from the following assumptions:

- Due to various materials involved in the composition of the crosssection, elasticity varies along with the height of the beam. As a consequence, cross-sectional rotation as implied by Bernoulli-Euler hypotheses does not take place about points laying in the plane passing through the centroid of the cross-section. Instead, these points are shifted along the $z$ axis by the (yet) unknown coordinate shift $\zeta_{0}$.

- Bending does take place in the $x-z$ plane and considered cross-sections are symmetric with respect to this plane in the geometrical and material sense. 
Therefore, a transverse coordinate $\zeta_{0}$ in which bending stresses disappear is now sought. The bending strain is described by $-w^{(2)}\left(z-\zeta_{0}\right)$, cf. Eq.(3). The bending strain is related to the stress by the non-local integral law Eq. (9)

$$
-w^{(2)} E_{i}\left(z-\zeta_{0}\right)=\int_{0}^{L} \phi_{\lambda, i}(x-\xi) \sigma_{\mathrm{b} i}(\xi, z) \mathrm{d} \xi=0
$$

where $\sigma_{\mathrm{b} i}$ denotes the bending stress in the $i$-th layer in which the neutral surface is positioned. The integral has to vanish due to assumption of the neutral surface in the layer $i$ at $\zeta_{0}$. Further, the left-hand side of the equation is integrated over the cross-section:

$$
0=\int_{0}^{A}-w^{(2)} E_{i}\left(z-\zeta_{0}\right) \mathrm{d} \bar{A} \Rightarrow 0=\sum_{i=1}^{n} b_{i} E_{i} \int_{z_{i-1}}^{z_{i}}\left(z-\zeta_{0}\right) \mathrm{d} z
$$

According to Box 3, $k_{E S}=\sum_{i=1}^{n} b_{i} E_{i} \int_{z_{i-1}}^{z_{i}}\left(z-\zeta_{0}\right) \mathrm{d} z=(\mathbf{E} \odot \mathbf{S}) \cdot \mathbf{1}=\mathbf{E} \cdot \mathbf{S}$, so $k_{E S}=0$. This result can be used to simplify equations obtained in previous sections, as well as to obtain the position of neutral surface. In particular:

$$
\sum_{i=1}^{n} b_{i} E_{i} \int_{z_{i-1}}^{z_{i}} z \mathrm{~d} z=\sum_{i=1}^{n} b_{i} E_{i} \int_{z_{i-1}}^{z_{i}} \zeta_{0} \mathrm{~d} z
$$

what gives the required shift of the neutral surface:

$$
\zeta_{0}=\frac{\sum_{i=1}^{n} A_{i} E_{i}\left(z_{i}+z_{i+1}\right)}{2 k_{E A}}
$$

The reader is cautioned that if Young modulus is a function of temperature, 
then the neutral surface shift is also temperature-dependent [51].

Aforementioned simplifications of governing equations (31, 35), boundary conditions Eqs. (32, 36, 37) and constitutive boundary conditions Eqs. (21. 22 due to $k_{E S}=0$ are summarized below for the reader's convenience:

$$
\begin{aligned}
& -k_{E A}^{2 N L} u_{0}^{(4)}+k_{E S}^{2 N L} w^{(5)}+k_{E A} u_{0}^{(2)}+k_{T N}^{(1)}+q_{x}=0 \\
& -k_{E S}^{2 N L} u_{0}^{(5)}+k_{E I}^{2 N L} w^{(6)}-k_{E I} w^{(4)}+k_{T M}^{(2)}+q_{z}=0 .
\end{aligned}
$$

Boundary conditions:

$$
\begin{array}{ll}
\left.\left(-k_{E A}^{2 N L} u_{0}^{(3)}+k_{E S}^{2 N L} w^{(4)}+k_{E A} u_{0}^{(1)}+k_{T N}\right)\right|_{x=0}=-\mathcal{N}_{0} & \text { or prescribe } u_{0}(0) \\
\left.\left(-k_{E A}^{2 N L} u_{0}^{(3)}+k_{E S}^{2 N L} w^{(4)}+k_{E A} u_{0}^{(1)}+k_{T N}\right)\right|_{x=L}=\mathcal{N}_{L} & \text { or prescribe } u_{0}(L) \\
\left.\left(-k_{E S}^{2 N L} u_{0}^{(3)}+k_{E I}^{2 N L} w^{(4)}-k_{E I} w^{(2)}+k_{T M}\right)\right|_{x=0}=-\mathcal{M}_{0} & \text { or prescribe } w^{(1)}(0) \\
\left.\left(-k_{E S}^{2 N L} u_{0}^{(3)}+k_{E I}^{2 N L} w^{(4)}-k_{E I} w^{(2)}+k_{T M}\right)\right|_{x=L}=\mathcal{M}_{L} & \text { or prescribe } w^{(1)}(L) \\
\left.\left(-k_{E S}^{2 N L} u_{0}^{(4)}+k_{E I}^{2 N L} w^{(5)}-k_{E I} w^{(3)}+k_{T M}^{(1)}\right)\right|_{x=0}=-\mathcal{T}_{0} & \text { or prescribe } w(0) \\
\left.\left(-k_{E S}^{2 N L} u_{0}^{(4)}+k_{E I}^{2 N L} w^{(5)}-k_{E I} w^{(3)}+k_{T M}^{(1)}\right)\right|_{x=L}=\mathcal{T}_{L} & \text { or prescribe } w(L) .
\end{array}
$$

Constitutive boundary conditions:

$$
\begin{aligned}
& \left(k_{E A}^{N L} u_{0}^{(2)}-k_{E S}^{N L} w^{(3)}-k_{T N}^{N L}\right)-\left(k_{E A} u_{0}^{(1)}-k_{2 T N}\right)=\left.0\right|_{x=0} \\
& \left(k_{E A}^{N L} u_{0}^{(2)}-k_{E S}^{N L} w^{(3)}-k_{T N}^{N L}\right)+\left(k_{E A} u_{0}^{(1)}-k_{2 T N}\right)=\left.0\right|_{x=L} \\
& \left(k_{E S}^{N L} u_{0}^{(2)}-k_{E I}^{N L} w^{(3)}-k_{T M}^{N L}\right)-\left(-k_{E I} w^{(2)}-k_{2 T M}\right)=\left.0\right|_{x=0} \\
& \left(k_{E S}^{N L} u_{0}^{(2)}-k_{E I}^{N L} w^{(3)}-k_{T M}^{N L}\right)+\left(-k_{E I} w^{(2)}-k_{2 T M}\right)=\left.0\right|_{x=L} .
\end{aligned}
$$

At the end, it is worth emphasizing that although $k_{E S}=0$, non-local coun- 
terparts do not vanish $k_{E S}^{N L}=\sum_{i=1}^{n} b_{i} E_{i} L_{\lambda, i} \int_{z_{i-1}}^{z_{i}}\left(z-\zeta_{0}\right) \mathrm{d} z \neq 0$ and $k_{E S}^{2 N L}=$ $\sum_{i=1}^{n} b_{i} E_{i} L_{\lambda, i}^{2} \int_{z_{i-1}}^{z_{i}}\left(z-\zeta_{0}\right) \mathrm{d} z \neq 0$ in general. Thus, coupling between axial and transverse displacements in both governing equations and boundary conditions exists. Decoupling is possible only in two special cases:

- Small size parameters $\lambda_{i}$ of all layers are equal. Since lengths of all layers in a beam are always equal to the beam's length $L$, then characteristic lengths of each layer $L_{\lambda, i}$ will be equal as well. This enables taking $L_{\lambda, i}$ and $L_{\lambda, i}^{2}$ out of summation signs and subsequent cancellation. Remaining terms are then equal to $k_{E S}$ and therefore vanish.

- Depending on particular coordinate values, integrals $\int_{z_{i-1}}^{z_{i}}\left(z-\zeta_{0}\right) \mathrm{d} z$ can be positive or negative. In a special case, positive values can balance negatives, leading to $k_{E S}^{N L}=0$ and $k_{E S}^{2 N L}=0$. This can take place in cross-sections symmetric about the $x-y$ coordinate plane with respect to geometrical and material properties, although some other possibilities also exist.

To summarize, coupling significantly complicates solution of the underlying system of 2 ordinary differential equations with 10 boundary conditions. Two possible solution procedures are described in the next section.

\section{Solution of the coupled problem}

Solution of the coupled non-homogenous ordinary differential problem Eqs. (42, 43, 44) is not straightforward. Nowadays, automated differential 
equations solvers like Mathematica, Matlab or alike are typically used for such purpose. In the present case, a direct approach by means of such software does not appear to have much success. Thus, two alternative solution strategies are considered below.

\subsection{Solution by substitution}

The first approach to solution of the problem Eqs. 42 with the boundary conditions Eqs. 43, 44) relies on the substitution:

$$
\bar{u}_{0}=u_{0}^{(2)}, \quad \bar{w}=w^{(4)}
$$

This reduces the order of the coupled system Eqs. (42) to

$$
\begin{aligned}
& -k_{E A}^{2 N L} \bar{u}_{0}^{(2)}+k_{E S}^{2 N L} \bar{w}^{(1)}+k_{E A} \bar{u}_{0}+k_{T N}^{(1)}+q_{x}=0 \\
& -k_{E S}^{2 N L} \bar{u}_{0}^{(3)}+k_{E I}^{2 N L} \bar{w}^{(2)}-k_{E I} \bar{w}+k_{T M}^{(2)}+q_{z}=0 .
\end{aligned}
$$

Upon solving the above coupled problem for $\bar{u}_{0}$ and $\bar{w}$, required displacement

fields follow from decoupled equations Eqs. (45). The solution will involve ten unknown integration constants that are solved from boundary conditions Eqs. 43, 44). Unfortunately, this approach seems to be suitable only for the simplest problems.

\subsection{Solution by Laplace transforms}

As a more efficient alternative to the substitution method, Laplace transforms are exploited. The central idea of Laplace transforms is to transform 
a problem involving ordinary differential equations into a problem based on algebraic equations. Although Laplace transforms are principally oriented toward initial value problems, the method can be successfully applied in the statical analysis of beams as well.

Firstly, Laplace transform is applied to the system of equations 42 giving:

$$
\begin{aligned}
& P_{u u}(s) U_{0}(s)+P_{u w}(s) W(s)+Q_{u}(s)=G_{u}(s), \\
& P_{w u}(s) U_{0}(s)+P_{w w}(s) W(s)+Q_{w}(s)=G_{w}(s)
\end{aligned}
$$

where

$$
\begin{aligned}
P_{u u}(s)= & k_{E A} s^{2}-k_{E A}^{2 N L} s^{4} \\
P_{u w}(s)= & k_{E S}^{2 N L} s^{5} W(s) \\
Q_{u}(s)= & k_{E A}^{2 N L} s^{3} u_{00}+k_{E A}^{2 N L} s^{2} u_{1}+k_{E A}^{2 N L} s u_{2}+k_{E A}^{2 N L} u_{3}-k_{E A} u_{00}-k_{E A} u_{1} \\
& -k_{E S}^{2 N L} s^{4} w_{0}-k_{E S}^{2 N L} s^{3} w_{1}-k_{E S}^{2 N L} s^{2} w_{2}-k_{E S}^{2 N L} s w_{3}-k_{E S}^{2 N L} w_{4} \\
P_{w w}(s)= & k_{E I} s^{4}-k_{E I}^{2 N L} s^{6} \\
P_{w u}(s)= & k_{E S}^{2 N L} s^{5} \\
Q_{w}(s)= & k_{E I}^{2 N L} s^{5} w_{0}+k_{E I}^{2 N L} s^{4} w_{1}+k_{E I}^{2 N L} s^{3} w_{2}+k_{E I}^{2 N L} s^{2} w_{3}+k_{E I}^{2 N L} s w_{4}+k_{E I}^{2 N L} w_{5} \\
& -k_{E I} s^{3} w_{0}-k_{E I} s^{2} w_{1}-k_{E I} s w_{2}-k_{E I} w_{3}-k_{E S}^{2 N L} s^{4} u_{00}-k_{E S}^{2 N L} s^{3} u_{1} \\
& -k_{E S}^{2 N L} s^{2} u_{2}-k_{E S}^{2 N L} s u_{3}-k_{E S}^{2 N L} u_{4}
\end{aligned}
$$


and Laplace transforms of corresponding functions are

$$
\begin{aligned}
U_{0}(s)=\mathcal{L}\left(u_{0}(x)\right), \quad W(s) & =\mathcal{L}(w(x)) \\
G_{u}(s)=\mathcal{L}\left(k_{T N}^{(1)}(x)+q_{x}(x)\right), \quad G_{w}(s) & =\mathcal{L}\left(k_{T M}^{(2)}(x)+q_{z}(x)\right) .
\end{aligned}
$$

Constants $u_{00}=u(0), u_{i}=u^{(i)}(0)$ and $w_{0}=w(0), w_{i}=w^{(i)}(0)$ denote initial conditions, in the present case displacement functions and their derivatives at the origin of the coordinate system $x=0$. These constants should be eventually determined from boundary and constitutive conditions Eqs. 43, 44). However, in the coupled problem at hand there are 11 initial conditions but 10 boundary and constitutive conditions, so the system seems to be underdetermined. The issue is circumvented by replacing two initial conditions terms associated with the highest order of derivatives with a single constant $u w_{45}=k_{E I}^{2 N L} w_{5}-k_{E S}^{2 N L} u_{4}$, thus reducing the number of initial conditions to 10. In the decoupled case described in Sec. $4.1 k_{E S}^{N L}=0$ and $k_{E S}^{2 N L}=0$, so $u w_{45}=k_{E I}^{2 N L} w_{5}$ reducing the number of integration constants by one and the latter substitution is not necessary.

Now, Laplace transforms $U_{0}(s)$ and $W(s)$ are easily obtained by the linear system of equations (47). With known $U_{0}(s)$ and $W(s)$ displacement functions follow from inverse Laplace transforms $u_{0}(x)=\mathcal{L}^{-1}\left(U_{0}(s)\right)$ and $w(x)=\mathcal{L}^{-1}(W(s))$. Although evaluation of inverse Laplace transform is straightforward, obtained terms are rather long thus being impractical for explicit documentation at this point. Finally, application of boundary and constitutive conditions Eqs. (43, 44) eliminates constants associated with 
initial conditions introduced above.

\section{Examples}

\subsection{Nonisothermal cantilever nanobeam in a homogeneous thermal field}

The introductory example considers a simple cantilever two-layer beam, free from external mechanical loading but situated in a homogeneous temperature field $\Delta \theta=0.1$. For present purposes, measurment units are deemed not to be important, so geometrical and material properties are assumed to be dimensionless. In that way, the unit length of the non-local beams is assumed $L=1$. Also: $b_{1}=b_{2}=1, h_{1}=h_{2}=1, E_{2}=2 E_{1}=2, \alpha_{2}=2 \alpha_{1}=0.2$.

The beam's cross-section is not symmetrical with respect to material properties. Thus, the neutral surface is shifted to the new position by $\zeta_{0}=1 / 6$.

Laplace transform of the system of differential equations is:

$$
\begin{gathered}
2 s^{5} W(s)-9 s^{4} U_{0}(s)+9 s^{2} u_{1}+300 s^{2} U_{0}(s)+9 s u_{2}+9 u_{3} \\
=2 s^{2} w_{2}+2 s w_{3}+300 u_{1}+2 w_{4}, \\
25 s^{6} W(s)-1100 s^{4} W(s)+24 s^{3} u_{1}+24 s^{2} u_{2}+24 s u_{3}+1100 s w_{2}+24 u_{4}+1100 w_{3} \\
=24 s^{5} U_{0}(s)+25 s^{3} w_{2}+25 s^{2} w_{3}+25 s w_{4}+25 w_{5} .
\end{gathered}
$$


Solving for $U_{0}(s)$ and $W(s)$ gives:

$$
\begin{aligned}
& U_{0}(s)=\frac{s\left(3\left(59 s^{2}-3300\right) u_{2}+177 s u_{3}+2 u w 45\right)+3\left(59 s^{4}-5800 s^{2}+110000\right) u_{1}+1100\left(2 w_{4}-9 u_{3}\right)}{3 s^{2}\left(59 s^{4}-5800 s^{2}+110000\right)}, \\
& W(s)=\frac{s\left(s\left(\left(59 s^{2}-5800\right)\left(s w_{2}+w_{3}\right)+59 s w_{4}+2400 u_{2}+3 u w_{45}\right)+100\left(24 u_{3}+1100 w_{2}-25 w_{4}\right)\right)-100\left(u w_{45}-1100 w_{3}\right)}{s^{4}\left(59 s^{4}-5800 s^{2}+110000\right)} .
\end{aligned}
$$

Peforming inverse Laplace transform gives required displacements but with unknown constants included:

$$
\begin{aligned}
u_{0}(x) & =e^{-13.59 x}\left(e^{18.66 x}\left(0.01255 u_{2}+0.002478 u_{3}-4.686 \cdot 10^{-6} u w_{45}-0.001017 w_{4}\right)\right. \\
& +e^{5.066 x}\left(0.002448 u_{2}-0.0002873 u_{3}+1.656 \cdot 10^{-6} u w_{45}-0.0002137 w_{4}\right) \\
& +e^{22.11 x}\left(0.002448 u_{2}+0.0002873 u_{3}+1.656 \cdot 10^{-6} u w_{45}+0.0002137 w_{4}\right) \\
& +e^{8.523 x}\left(0.01255 u_{2}-0.002478 u_{3}-4.686 \cdot 10^{-6} u w_{45}+0.001017 w_{4}\right) \\
& \left.+e^{13.59 x}\left(-0.03 u_{2}+6.061 \cdot 10^{-6} u w_{45}+u_{1} x-0.03 u_{3} x+0.006667 w_{4} x\right)\right) \\
w(x) \quad & =e^{-13.59 x}\left(e^{5.066 x}\left(-0.0006994 u_{2}+0.00008207 u_{3}-\left(4.731 \cdot 10^{-7}\right) u w_{45}+0.00006106 w_{4}\right)\right. \\
& +e^{22.11 x}\left(0.0006994 u_{2}+0.00008207 u_{3}+4.731 \cdot 10^{-7} u w_{45}+0.00006106 w_{4}\right) \\
& +e^{8.523 x}\left(0.00333 u_{2}-0.0006573 u_{3}-1.243 \cdot 10^{-6} u w_{45}+0.0002699 w_{4}\right) \\
& +e^{18.66 x}\left(-0.00333 u_{2}-0.0006573 u_{3}+1.243 \cdot 10^{-6} u w_{45}+0.0002699 w_{4}\right) \\
& +e^{13.59 x}\left(w_{4}\left(-0.01136 x^{2}-0.000662\right)+u_{3}\left(0.01091 x^{2}+0.00115\right)\right. \\
& \left.\left.+x\left(-0.0001515 u w_{45} x^{2}+0.1667 w_{3} x^{2}+0.5 w_{2} x+0.02182 u_{2}-0.00002066 u w_{45}\right)\right)\right)
\end{aligned}
$$

Constants follow from solving the nonlinear system of algebraic equations:

$$
\begin{gathered}
u_{1}=-0.01659, u_{2}=0.0004134, u_{3}=0.002107, u w_{45}-0.5818, \\
w_{2}=0.007167, w_{3}=-0.0005289, w_{4}=-0.002614,
\end{gathered}
$$


so final results are:

$$
\begin{aligned}
u_{0}(x) & =-0.01667 x+1.981 \cdot 10^{-9} e^{-8.523 x}+3.405 \cdot 10^{-8} e^{-5.066 x} \\
& +0.0000158 e^{5.066 x}+9.536 \cdot 10^{-8} e^{8.523 x}-0.00001593 \\
w(x) & =-2.822 \cdot 10^{-14} x^{3}+0.003636 x^{2}+0.00002104 x-5.66 \cdot 10^{-10} e^{-8.523 x} \\
& +9.034 \cdot 10^{-9} e^{-5.066 x}-4.191 \cdot 10^{-6} e^{5.066 x} \\
& +2.724 \cdot 10^{-8} e^{8.523 x}+4.155 \cdot 10^{-6}
\end{aligned}
$$

The layered structure also causes bending of the beam in local and nonlocal cases alike. The non-local case is illustrated in Fig. 1. The uniform axial elongation observed in local beams is also affected and a nonlinear pattern is visible at the free end of the beam, Fig. 2

It is well-known that homogeneous beams subjected to a constant (or linear) temperature field do not give rise to stresses [69 71]. However, in the case of layered beams, the issue is somewhat more complex. To illustrate this behavior, the strain field $\varepsilon$ is given in Fig. 3. The strain field is smooth, without discontinuities caused by the layered structure. This is not the case with the stress field, Fig. 6. To point out the non-local character of the problem, local and non-local parts of the stress field (Eq. 10) are also shown, Figs. 4, 5. Such discontinuities can be attributed to differences in material properties. The magnitude of the local part is higher than the non-local part; this obviously depends on particular values of non-local parameters. 


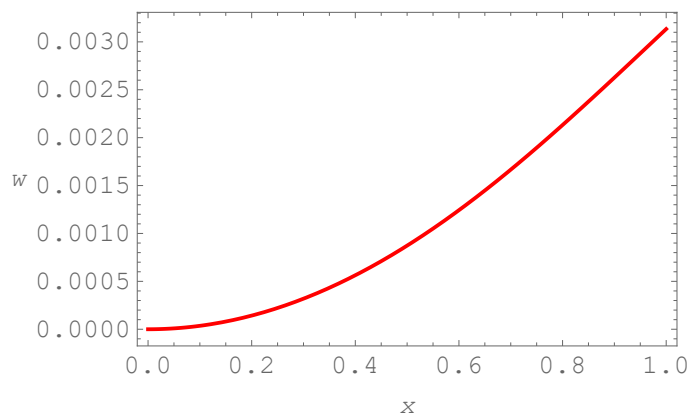

Figure 1: Transverse displacements of the beam in the homogeneous temperature field.

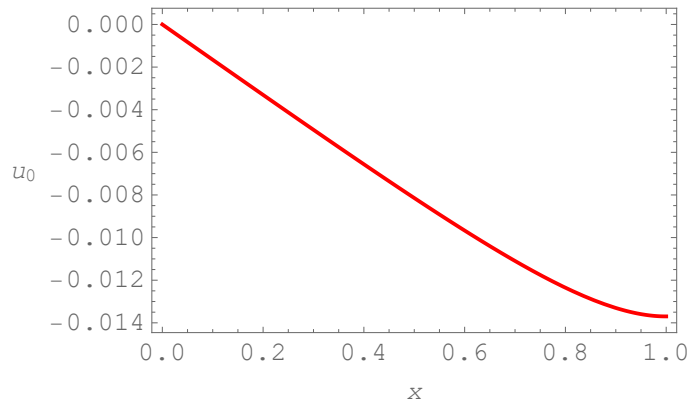

Figure 2: Axial displacements of the beam in the homogeneous temperature field.

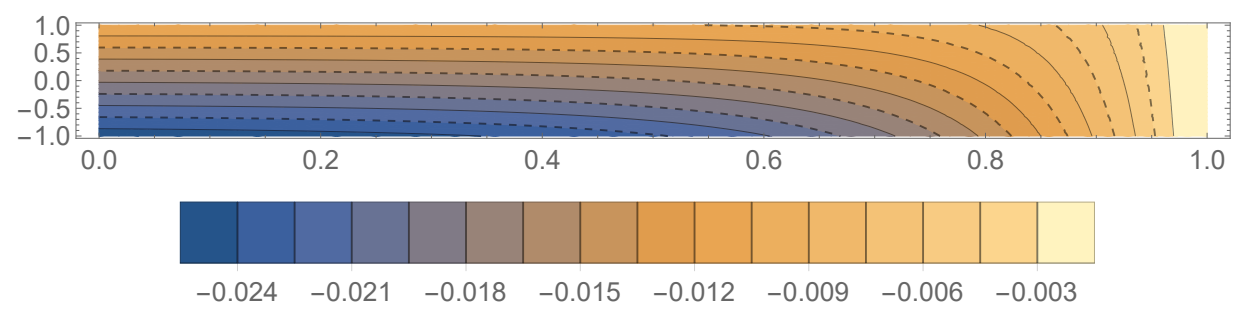

Figure 3: Normal strain distribution in the beam loaded by the homogeneous temperature field. 


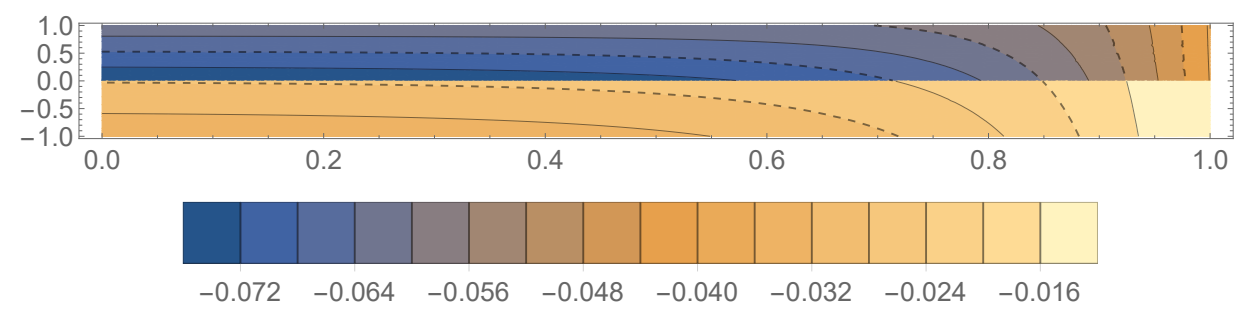

Figure 4: Local part of the stress field in the beam in the homogeneous temperature field.

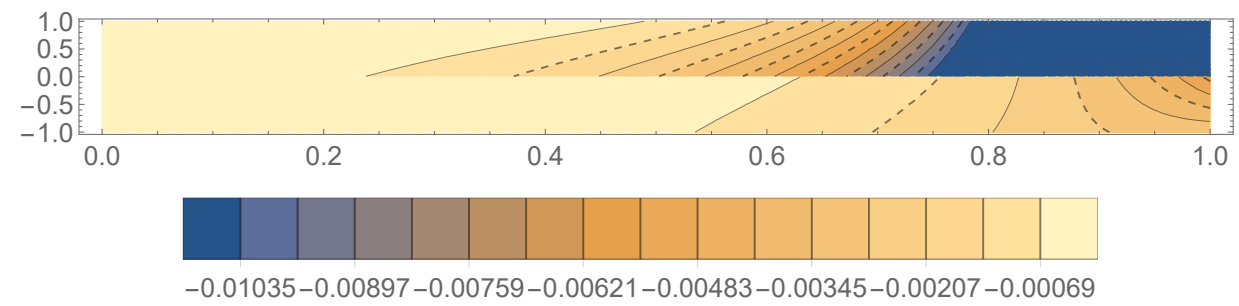

Figure 5: Non-local part of the stress field in the beam in the homogeneous temperature field.

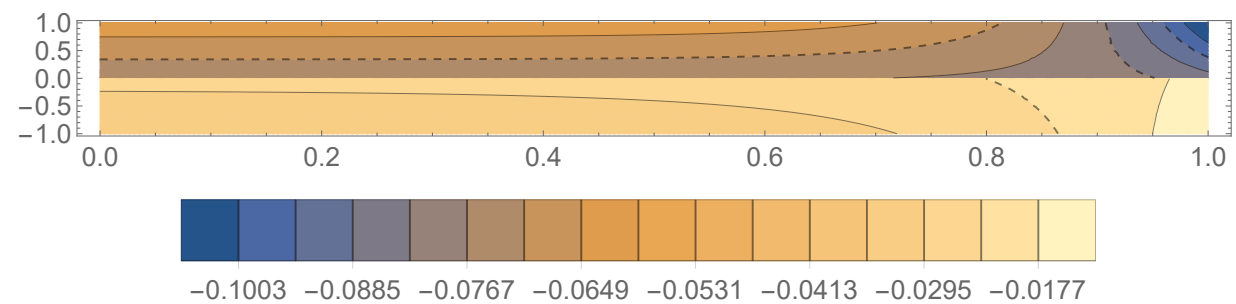

Figure 6: Total stress field (local + non-local) in the beam in the homogeneous temperature field. 


\subsection{Nonisothermal cantilever piezoelectric nanobeam}

The second example considers a two-layer piezoelectric cantilever beam, Fig. 7. The beam is subjected to a linear temperature variation in the transverse direction $\Delta \theta(z)=0.1 z$. In addition to the temperature load, two different mechanical loads will be separately considered:

- axial force at the tip $F_{x}$ and

- transverse force at the tip $F_{x}$.

The beam is composed of two layers: the first one is $\mathrm{SiO}_{2}$ and the second one $\mathrm{Pb}\left(\mathrm{Zr}_{0.4} \mathrm{Ti}_{0.6}\right) \mathrm{O}_{3}$ (PZT 40/60). Material data is taken from [72]: the Young's modulus of $\mathrm{SiO}_{2}$ is $E_{1}=77 \mathrm{GPa}$ and the coefficient of thermal exapnsion is $\alpha_{1}=5.5 \times 10^{-7} 1 / \mathrm{K}$. For the PZT $40 / 60$ these material parameters are $E_{2}=85 \mathrm{GPa}$ and $\alpha_{2}=3.7 \times 10^{-6} 1 / \mathrm{K}$. Thicknesses of each layer are $h_{1}=600$ $\mathrm{nm}$ and $h_{2}=200 \mathrm{~nm}$. Width of beam's layers is $b=1200 \mathrm{~nm}$ while the beam's length is $L=15000 \mathrm{~nm}$. It is necessary to determine variation of coupling terms $k_{E S}^{N L}$ and $k_{E S}^{2 N L}$ with respect to non-local parameters $\lambda_{1}$ and $\lambda_{2}$. For the specific choice of $\lambda_{1}=0.1$ and $\lambda_{2}=0.2$, calculate displacement fields caused by axial force and transverse forces in the range $F_{x}, F_{z} \in[0,10000]$ $\mathrm{nN}$.

Fig. 8 presents variations in coupling terms that stem from the first moment of area. It is clearly visible that for $\lambda_{1}=\lambda_{2}$ both terms vanish, thus decoupling the problem. For all other choices of small-size parameters and given geometry and material composition, displacement fields are coupled. 


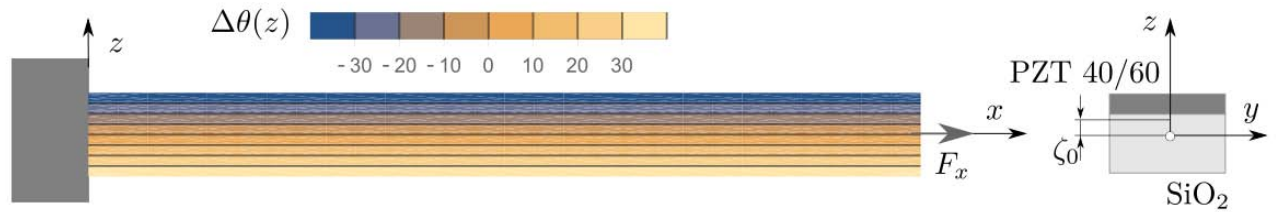

Figure 7: Cantilever beam loaded by the axial force $F_{x}$ and temperature field $\Delta \theta(z)$.
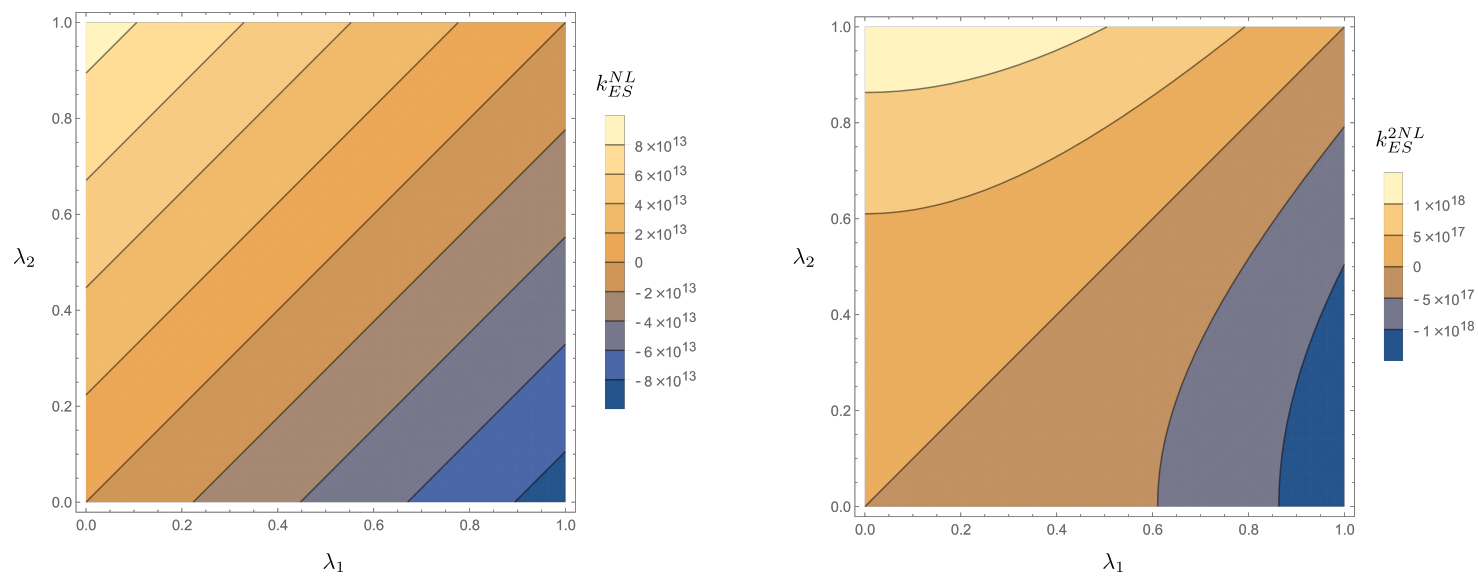

Figure 8: Variation of coupling terms $k_{E S}^{N L}\left(\mathrm{nN} \cdot \mathrm{nm}^{2}\right)$ and $k_{E S}^{2 N L}\left(\mathrm{nN} \cdot \mathrm{nm}^{3}\right)$ vs. non-local parameters $\lambda_{1}$ and $\lambda_{2}$ 

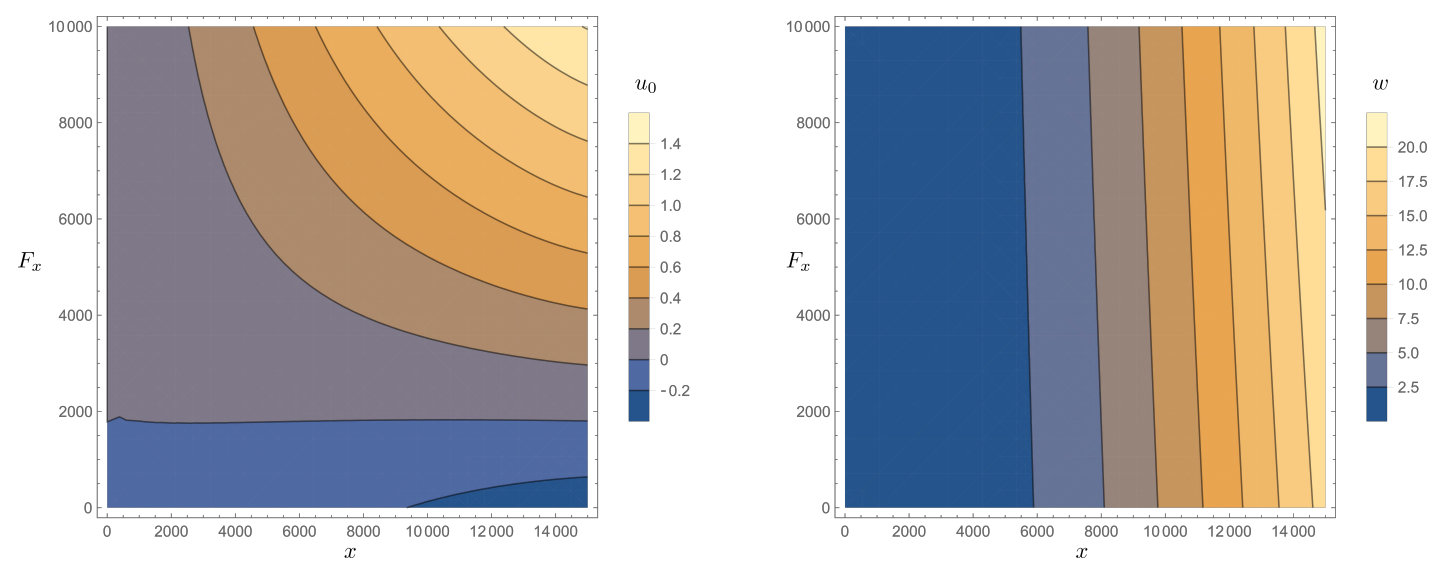

Figure 9: Variations of the axial displacement $u_{0}(\mathrm{~nm})$ and transverse displacement $w$ $(\mathrm{nm})$ along the beam's length $x(\mathrm{~nm})$ vs. axial force $F_{x}(\mathrm{nN})$

\subsubsection{Axial force and temperature field $\Delta \theta(z)$}

Distribution of the axial displacement and transverse displacement along the beam for different values of the axial force $F_{x}$ is given in Fig. 9. Observe that even for the tensile axial force up to approximately $2000 \mathrm{nN}$ the negative axial displacement for the complete beam is obtained. This kind of behavior is caused by coupling between axial and transverse displacements. The transverse displacement remains positive for all choices of the axial force. The influence of the axial displacement on the transverse displacement field thus remains small and the bending is caused by the temperature field.

\subsubsection{Transverse force and temperature field $\Delta \theta(z)$}

In the case of transverse load, for the specific choice of $F_{z}=1000 \mathrm{nN}$ distributions of both displacements are provided in Figs. 10 and 11. Again, Fig. 12 illustrates the variation of axial and transverse displacements for different transverse forces and given temperature field. Bending behavior 


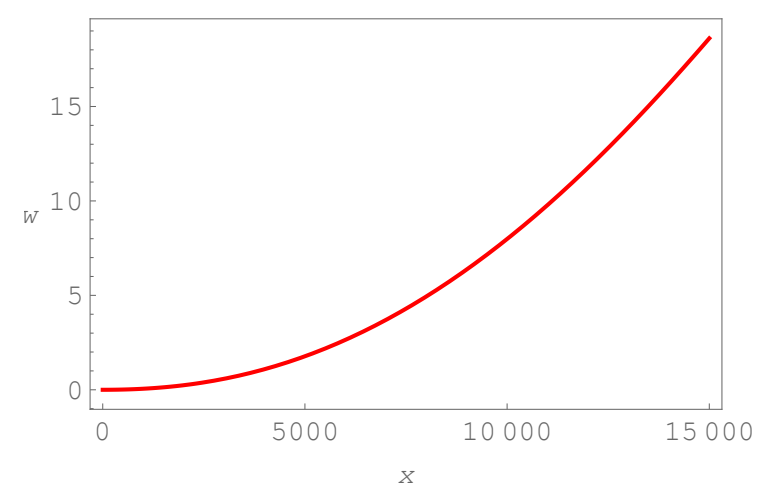

Figure 10: Transverse displacements of the beam for $F_{z}=1000 \mathrm{nN}$ and $\Delta \theta(z)$.

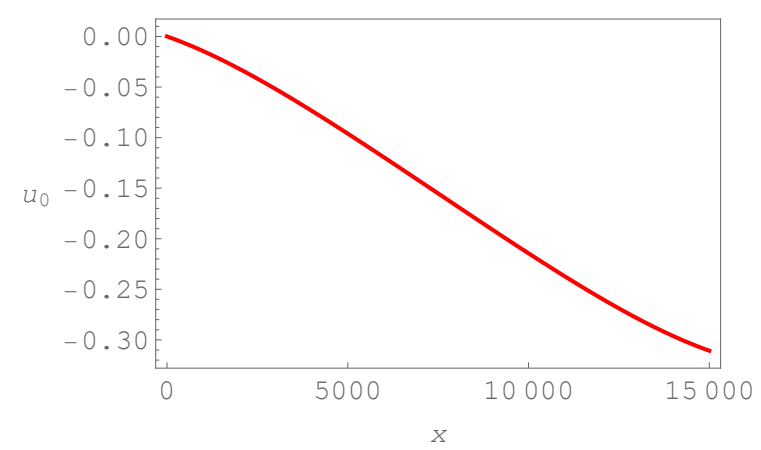

Figure 11: Axial displacements of the beam for $F_{z}=1000 \mathrm{nN}$ and $\Delta \theta(z)$.

clearly dominates over much smaller axial displacements.

\subsection{Thermally and mechanically loaded doubly clamped nanobeam}

The present example aims to demonstrate advantage of Laplace transforms approach in order to solve a beam loaded by nonlinear mechanical and thermal loads. Kinematically, the beam is clamped at both ends and thus statically indeterminate, Fig. 13 . The transverse mechanical load is distributed in a parabolic manner $q_{z}(x)=\frac{1}{4}-\left(x-\frac{L}{2}\right)^{2}$, while the temperature change is governed by the sinus function $\Delta \theta(x)=100 \sin \left(2 \pi \frac{x}{L}\right)$. The beam 

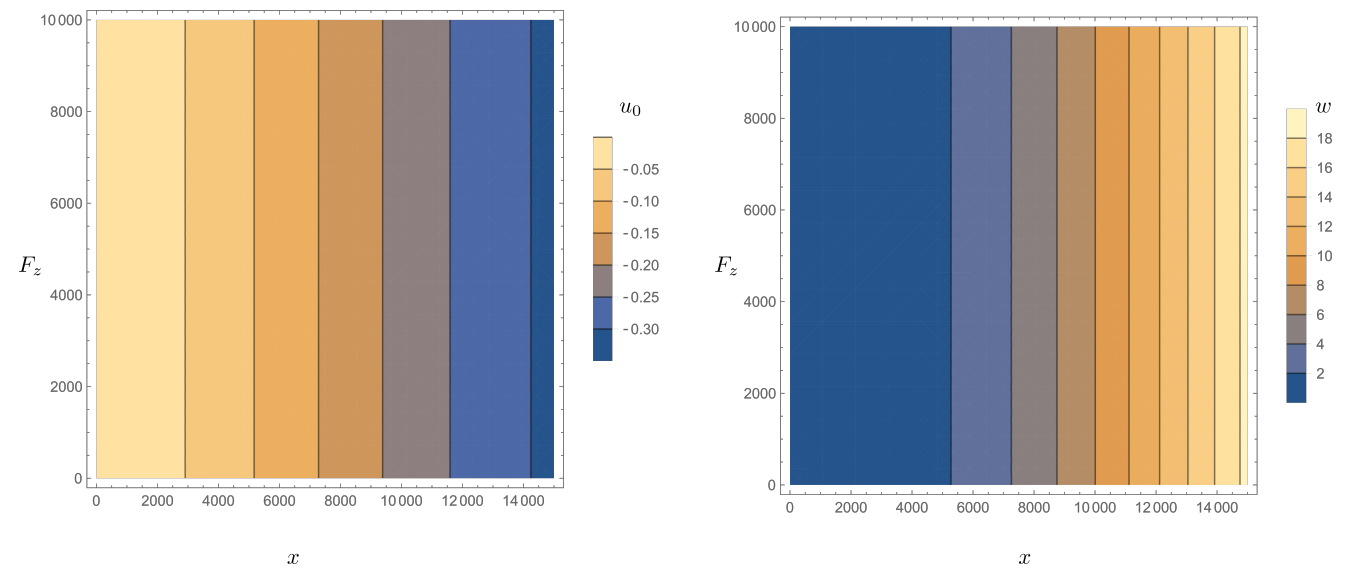

Figure 12: Variations of the axial displacement $u_{0}(\mathrm{~nm})$ and transverse displacement $w$ $(\mathrm{nm})$ along the beam's length $x(\mathrm{~nm})$ vs. transverse force $F_{z}(\mathrm{nN})$

is assumed to be composed of two layers with following properties

$$
\begin{gathered}
\mathbf{b}=\left[\begin{array}{l}
1 \\
3
\end{array}\right], \quad \mathbf{h}=\left[\begin{array}{l}
3 \\
1
\end{array}\right], \\
\mathbf{E}=\left[\begin{array}{l}
1 \\
2
\end{array}\right], \quad \boldsymbol{\alpha}=\left[\begin{array}{l}
0.1 \\
0.2
\end{array}\right], \quad \boldsymbol{\lambda}=\left[\begin{array}{l}
0.1 \\
0.2
\end{array}\right] .
\end{gathered}
$$

Thus, the beam has $\mathrm{T}$ shaped cross-section. The beam is of unit length $L=1$.

This shift of the neutral surface is readiliy evaluated as $\zeta_{0}=1 / 3$.

The system of ordinary differential equations Eq. 42 is now restated by 


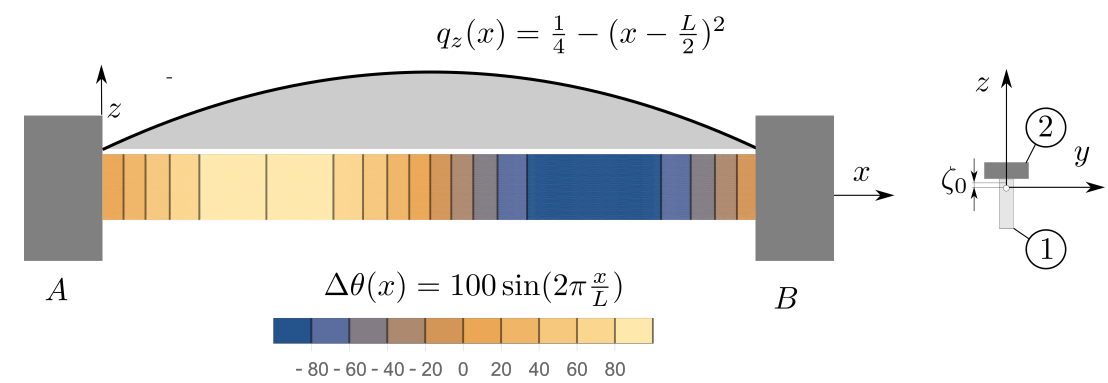

Figure 13: Doubly clamped beam loaded by the distributed parabolic load $q_{z}(x)$ and sinusoidal temperature field $\Delta \theta(x)$.

aid of Laplace transforms in the manner of Eqs. (47) as:

$$
\begin{aligned}
& \frac{3}{25} s^{5} W(s)-\frac{27}{100} s^{4} U_{0}(s)-\frac{3 s^{4} w_{0}}{25}+\frac{27 s^{3} u_{00}}{100}-\frac{3 s^{3} w_{1}}{25}+\frac{27 s^{2} u_{1}}{100} \\
& +9 s^{2} U_{0}(s)-\frac{3 s^{2} w_{2}}{25}+\frac{300 \pi s}{s^{2}+4 \pi^{2}}+\frac{27 s u_{2}}{100}-\frac{3 s w_{3}}{25}+\frac{27 u_{3}}{100}-\frac{3 w_{4}}{25}-9 s u_{00}-9 u_{1}=0 \\
& \frac{81}{400} s^{6} W(s)-\frac{3}{25} s^{5} U_{0}(s)-\frac{81 s^{5} w_{0}}{400}+\frac{3 s^{4} u_{00}}{25}-\frac{81 s^{4} w_{1}}{400}-\frac{43}{4} s^{4} W(s) \\
& +\frac{3 s^{3} u_{1}}{25}+\frac{43 s^{3} w_{0}}{4}-\frac{81 s^{3} w_{2}}{400}+\frac{3 s^{2} u_{2}}{25}+\frac{43 s^{2} w_{1}}{4}-\frac{81 s^{2} w_{3}}{400}+\frac{1}{s^{2}}+\frac{3 s u_{3}}{25} \\
& +\frac{43 s w_{2}}{4}-\frac{81 s w_{4}}{400}+\frac{3 u_{4}}{25}+\frac{43 w_{3}}{4}-\frac{81 w_{5}}{400}-\frac{2}{s^{3}}-\frac{320 \pi^{3}}{s^{2}+4 \pi^{2}}=0 .
\end{aligned}
$$

Replacing two integration constants with a single one $u w_{45}=\frac{3 u_{4}}{25}-\frac{81 w_{5}}{400}$ and 
solving for $U_{0}(s), W(s)$ provides

$$
\begin{aligned}
& U_{0}(s)=-\frac{1}{3 s^{4}\left(s^{2}+4 \pi^{2}\right)\left(179 s^{4}-21000 s^{2}+430000\right)}\left(-537 s^{9} u_{00}-537 s^{8} u_{1}-2148 \pi^{2} s^{7} u_{00}\right. \\
& +63000 s^{7} u_{00}-537 s^{7} u_{2}-2148 \pi^{2} s^{6} u_{1}+63000 s^{6} u_{1}-537 s^{6} u_{3}+252000 \pi^{2} s^{5} u_{00} \\
& -1290000 s^{5} u_{00}-2148 \pi^{2} s^{5} u_{2}+38700 s^{5} u_{2}+1600 s^{5} u w_{45}-810000 \pi s^{5} \\
& +252000 \pi^{2} s^{4} u_{1}-1290000 s^{4} u_{1}-2148 \pi^{2} s^{4} u_{3}+38700 s^{4} u_{3}-17200 s^{4} w_{4} \\
& -5160000 \pi^{2} s^{3} u_{00}+154800 \pi^{2} s^{3} u_{2}+6400 \pi^{2} s^{3} u w_{45}-512000 \pi^{3} s^{3} \\
& +43000000 \pi s^{3}+1600 s^{3}-5160000 \pi^{2} s^{2} u_{1}+154800 \pi^{2} s^{2} u_{3}-68800 \pi^{2} s^{2} w_{4} \\
& \left.-3200 s^{2}+6400 \pi^{2} s-12800 \pi^{2}\right)
\end{aligned}
$$

and

$$
\begin{aligned}
& W(s)=-\frac{1}{s^{7}\left(s^{2}+4 \pi^{2}\right)\left(179 s^{4}-21000 s^{2}+43000\right)}\left(-179 s^{12} w_{0}-179 s^{11} w_{1}-716 \pi^{2} s^{10} w_{0}\right. \\
& +21000 s^{10} w_{0}-179 s^{10} w_{2}-716 \pi^{2} s^{9} w_{1}+21000 s^{9} w_{1}-179 s^{9} w_{3} \\
& +84000 \pi^{2} s^{8} w_{0}-430000 s^{8} w_{0}-716 \pi^{2} s^{8} w_{2}+21000 s^{8} w_{2}-179 s^{8} w_{4} \\
& -4800 s^{7} u_{2}+1200 s^{7} u w_{45}+84000 \pi^{2} s^{7} w_{1}-430000 s^{7} w_{1}-716 \pi^{2} s^{7} w_{3} \\
& +21000 s^{7} w_{3}-160000 \pi s^{7}-4800 s^{6} u_{3}-1720000 \pi^{2} s^{6} w_{0}+84000 \pi^{2} s^{6} w_{2} \\
& -430000 s^{6} w_{2}-716 \pi^{2} s^{6} w_{4}+8100 s^{6} w_{4}-19200 \pi^{2} s^{5} u_{2}+4800 \pi^{2} s^{5} u w_{45} \\
& -40000 s^{5} u w_{45}-1720000 \pi^{2} s^{5} w_{1}+84000 \pi^{2} s^{5} w_{3}-430000 s^{5} w_{3} \\
& -384000 \pi^{3} s^{5}+1200 s^{5}-19200 \pi^{2} s^{4} u_{3}-1720000 \pi^{2} s^{4} w_{2}+32400 \pi^{2} s^{4} w_{4} \\
& -2400 s^{4}-160000 \pi^{2} s^{3} u_{w} 45-1720000 \pi^{2} s^{3} w_{3}+12800000 \pi^{3} s^{3}+4800 \pi^{2} s^{3} \\
& \left.-40000 s^{3}-9600 \pi^{2} s^{2}+80000 s^{2}-160000 \pi^{2} s+320000 \pi^{2}\right)
\end{aligned}
$$

Finally, inverse Laplace transforms $\mathcal{L}^{-1}\left(U_{0}(s)\right)$ and $\mathcal{L}^{-1}(W(s))$ give the re- 
quired displacement functions that include unknown constants and support reactions. These are determined from boundary conditions Eq. (43) and constitutive boundary conditions Eq. (44). In the present case there is

$$
\begin{aligned}
u_{0}(0)= & u_{0}(L)=w(0)=w(L)=0, w^{(1)}(0)=w^{(1)}(L)=0 \text { and: } \\
& \left.\left(-k_{E A}^{2 N L} u_{0}^{(3)}+k_{E S}^{2 N L} w^{(4)}+k_{E A} u_{0}^{(1)}+k_{T N}\right)\right|_{x=L}=\mathcal{N}_{L} \\
& \left.\left(-k_{E S}^{2 N L} u_{0}^{(3)}+k_{E I}^{2 N L} w^{(4)}-k_{E I} w^{(2)}+k_{T M}\right)\right|_{x=L}=\mathcal{M}_{L} \\
& \left.\left(-k_{E S}^{2 N L} u_{0}^{(4)}+k_{E I}^{2 N L} w^{(5)}-k_{E I} w^{(3)}+k_{T M}^{(1)}\right)\right|_{x=L}=\mathcal{T}_{L} \\
& \left(k_{E A}^{N L} u_{0}^{(2)}-k_{E S}^{N L} w^{(3)}-k_{T N}^{N L}\right)-\left(k_{E A} u_{0}^{(1)}-k_{2 T N}\right)=\left.0\right|_{x=0} \\
& \left(k_{E A}^{N L} u_{0}^{(2)}-k_{E S}^{N L} w^{(3)}-k_{T N}^{N L}\right)+\left(k_{E A} u_{0}^{(1)}-k_{2 T N}\right)=\left.0\right|_{x=L} \\
& \left(k_{E S}^{N L} u_{0}^{(2)}-k_{E I}^{N L} w^{(3)}-k_{T M}^{N L}\right)-\left(-k_{E I} w^{(2)}-k_{2 T M}\right)=\left.0\right|_{x=0} \\
& \left(k_{E S}^{N L} u_{0}^{(2)}-k_{E I}^{N L} w^{(3)}-k_{T M}^{N L}\right)+\left(-k_{E I} w^{(2)}-k_{2 T M}\right)=\left.0\right|_{x=L}
\end{aligned}
$$

where $\mathcal{N}_{L}, \mathcal{M}_{L}$ and $\mathcal{T}_{L}$ are unknown support reactions at $x=L$. The algebraic system of equations is solved for unknown constants and support reactions:

$$
\begin{gathered}
u_{00}=0, u_{1}=-8.096, u_{2}=-16.57, u_{3}=-49.26, u w_{45}=959.8 \\
w_{0}=0, w_{1}=0, w_{2}=3.332, w_{3}=-52.77, w_{4}=496.4 \\
\mathcal{N}_{L}=-5.196 \cdot 10^{-5}, \mathcal{M}_{L}=-392.7, \mathcal{T}_{L}=-70.65 .
\end{gathered}
$$


Final forms of displacement distributions can be finally written as:

$$
\begin{aligned}
u_{0}(x)= & 4.134 \cdot 10^{-4} x^{3}-6.202 \cdot 10^{-4} x^{2}+1.154 \cdot 10^{-4} x-3.034 \\
& -0.268 e^{-9.534 x}-1.941 \cdot 10^{-5} e^{9.534 x}+2.084 e^{-5.141 x} \\
& +1.212 \cdot 10^{-2} e^{5.141 x}+1.206 \cos (6.283 x)
\end{aligned}
$$

and

$$
\begin{aligned}
w(x)= & -2.584 \cdot 10^{-4} x^{6}+7.752 \cdot 10^{-4} x^{5}-1.460 \cdot 10^{-4} x^{4}+2.189 x^{3} \\
& -3.284 x^{2}+1.649 x-0.277 \\
& +4.007 \cdot 10^{-2} e^{-9.534 x}-2.902 \cdot 10^{-6} e^{9.534 x}+0.238 e^{-5.141 x} \\
& -1.394 \cdot 10^{-3} e^{5.141 x}-5.545 \cdot 10^{-3} \sin (6.283 x) .
\end{aligned}
$$

Displacements are shown in Figs. 14 and 15. As expected, the U-shaped curve typical for transverse displacements of homogeneous local beams is not obtained. This can be attributed to complex interaction of the loading, coupling terms and layer distribution of the non-local beam. Strains arising from both mechanical and thermal loads, Fig. 16 show a similar order of magnitude. Discontinuities are absent if the cumulative strain is considered, but not if only mechanical or only thermal strain is concerned. Thus, combination of two discontinuous fields gives a continuous one. In the case of stress field, discontinuities are also present in the total stress field. Higher stresses are obtained in the thinner layer. 


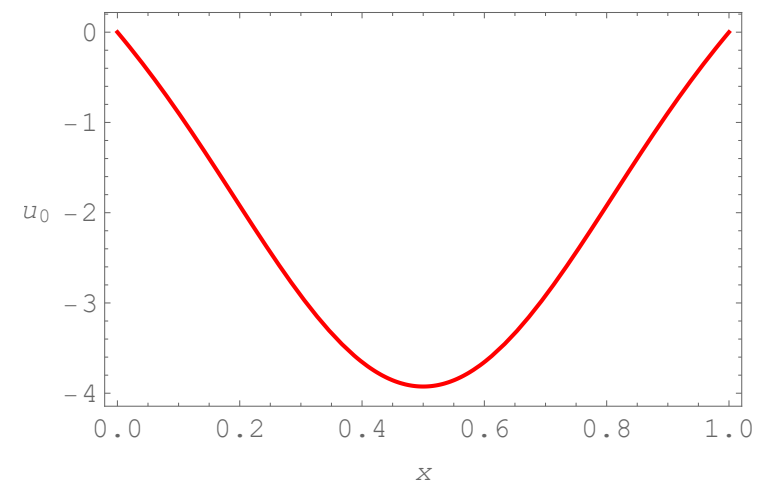

Figure 14: Axial displacements of the doubly clamped beam.

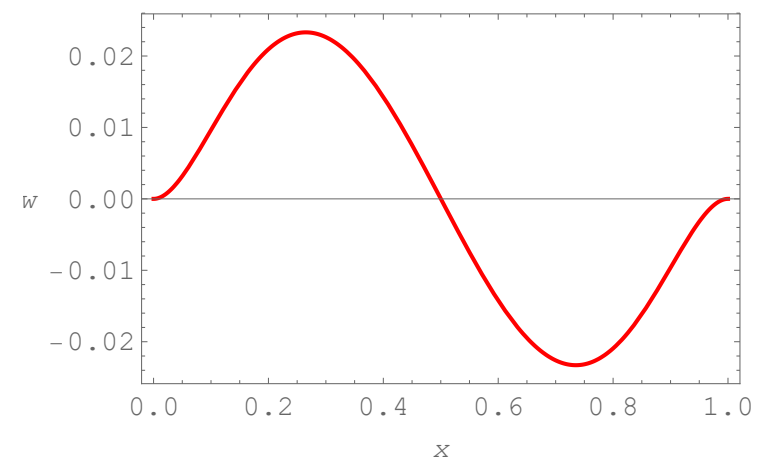

Figure 15: Transversal displacements of the doubly clamped beam. 

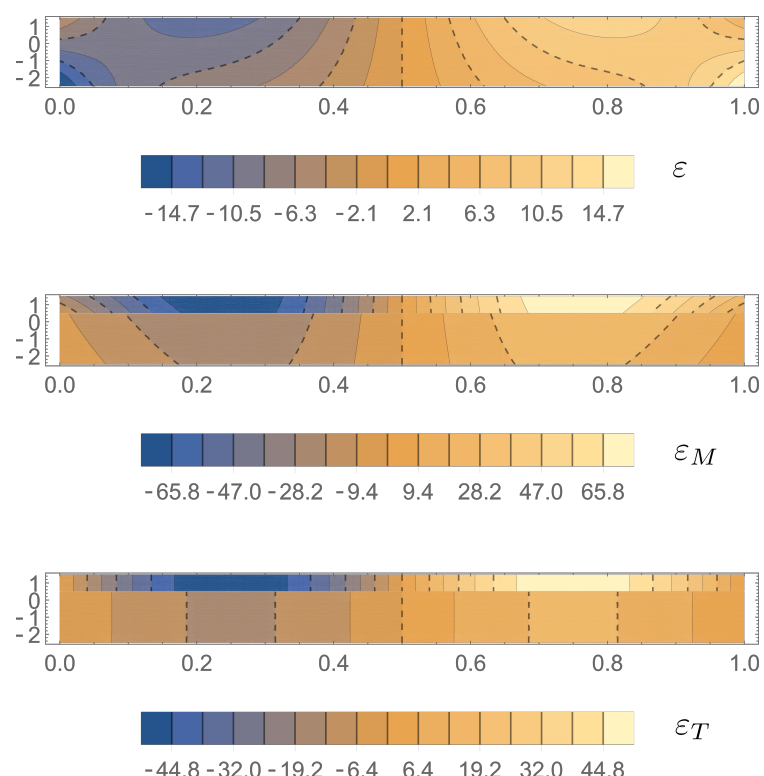

Figure 16: Strain distribution in the doubly clamped beam.
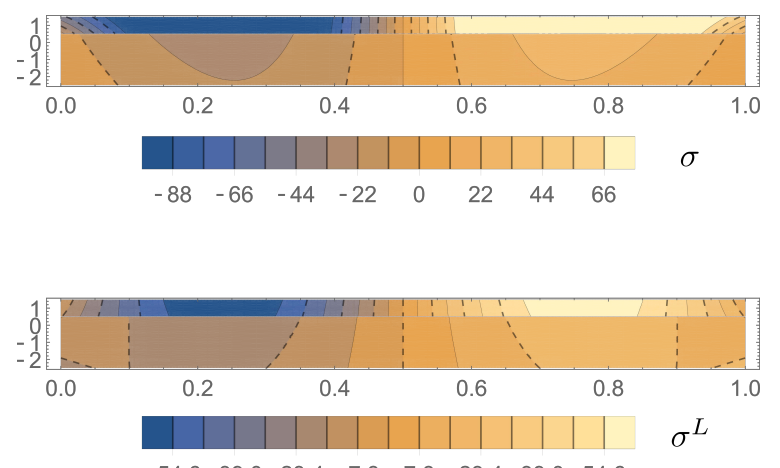

$\begin{array}{llllll}-54.6-39.0-23.4 & -7.8 & 7.8 & 23.4 & 39.0 & 54.6\end{array}$

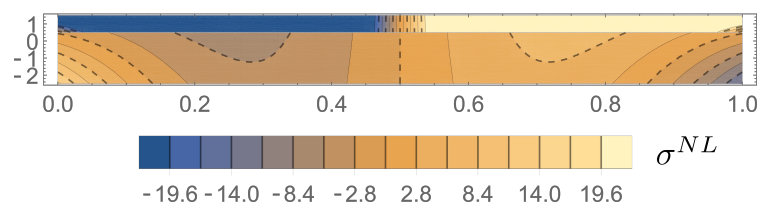

Figure 17: Total stress $\sigma=\sigma^{L}+\sigma^{N L}$, local part $\sigma^{L}$ of the stress and non-local part of the stress $\sigma^{N L}$ in a doubly clamped beam. 


\subsection{Influence of distribution of layers}

To evaluate different possibilities of layers' arrangement and their influence on mechanical behavior, a simply supported beam made of 4 layers is considered in the final example. Two different materials are used for layers and four different layered structures were considered. These are described by four-digits codes as 1122, 1221, 1212, 2112, where each digit denotes material ID, either 1 or 2, see Fig. 18. Material data is again considered in non-dimensional form as $E_{1}=100, E_{2}=200, \alpha_{1}=0.1, \alpha_{2}=0.2$, $\lambda_{1}=0.1, \lambda_{2}=0.2$. The beam's width is constant $b=3$, while the height of each layer is $h_{i}=1$, where $i=1,2,3,4$. In that way the total height of the beam is $h=\sum_{i} h_{i}=4$. The beam's length is $L=100$. Beam is mechanically loaded with the distributed loading in both longituidnal $q_{x}=1$ and transverse direction $q_{z}=1$. As for the thermal loading, two cases are considered, Fig. 18:

(a) $\Delta \theta(x)=10 \frac{x}{L}$ and

(b) $\Delta \theta(x, z)=10 \frac{x}{L}+10 \frac{z}{h / 2}$.

The system of ordinary differential equations Eq. (47) is solved by Laplace transforms as before, with constitutive boundary conditions Eqs. (44) and 

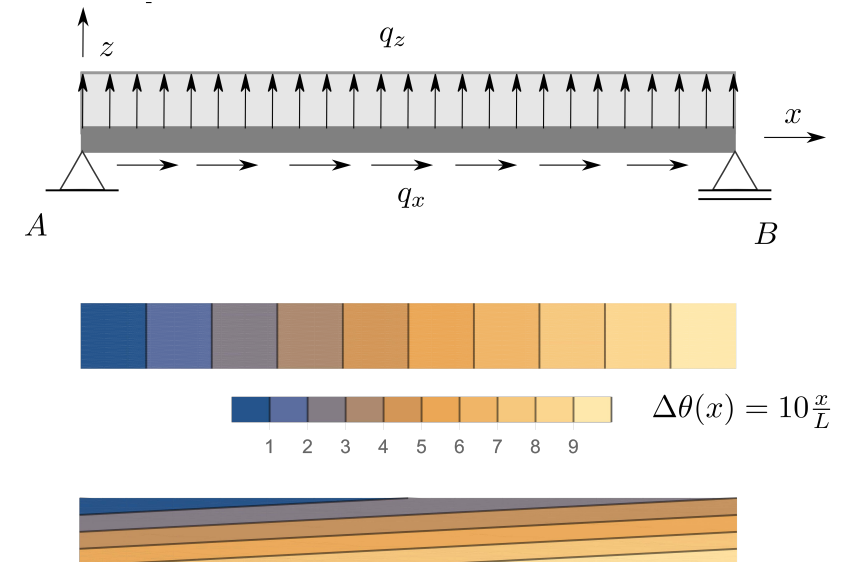

$|+||| \quad|\quad| \quad|\quad| \quad|\quad| z, z)=10 \frac{x}{L}+10 \frac{z}{h / 2}$
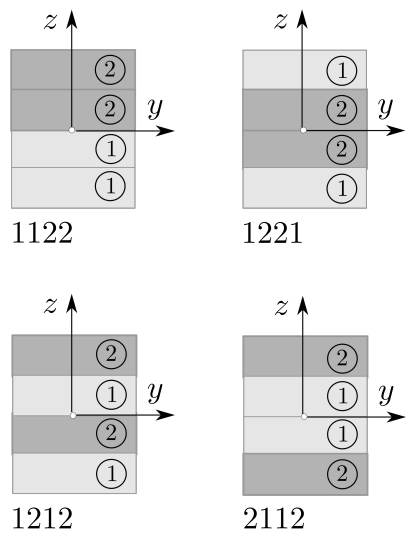

Figure 18: Simply supported beam and considered layered structures.

boundary conditions Eqs. 43 as $u_{0}(0)=w(0)=w(L)=0$ and

$$
\begin{aligned}
& \left.\left(-k_{E A}^{2 N L} u_{0}^{(3)}+k_{E S}^{2 N L} w^{(4)}+k_{E A} u_{0}^{(1)}+k_{T N}\right)\right|_{x=L}=0 \\
& \left.\left(-k_{E S}^{2 N L} u_{0}^{(3)}+k_{E I}^{2 N L} w^{(4)}-k_{E I} w^{(2)}+k_{T M}\right)\right|_{x=0}=0 \\
& \left.\left(-k_{E S}^{2 N L} u_{0}^{(3)}+k_{E I}^{2 N L} w^{(4)}-k_{E I} w^{(2)}+k_{T M}\right)\right|_{x=L}=0
\end{aligned}
$$

Solutions are graphically presented in Figs. 19 - 22. Symmetric layer distributions 1221 and 2112 lead toward vanishing of the shift of the neutral surface. In these two cases terms $k_{E S}^{N L}=0$ and $k_{E S}^{2 N L}=0$ so the problem is decoupled. In the Case (a), temperature does not change in the transverse direction. Nevertheless, the influence of different layer distributions can be noticed. Albeit the influence is small in the case of axial displacements, Fig. 19, transverse displacements are markedly different, Fig. 20. Since the temperature does not change along the transverse coordinate, such behavior 


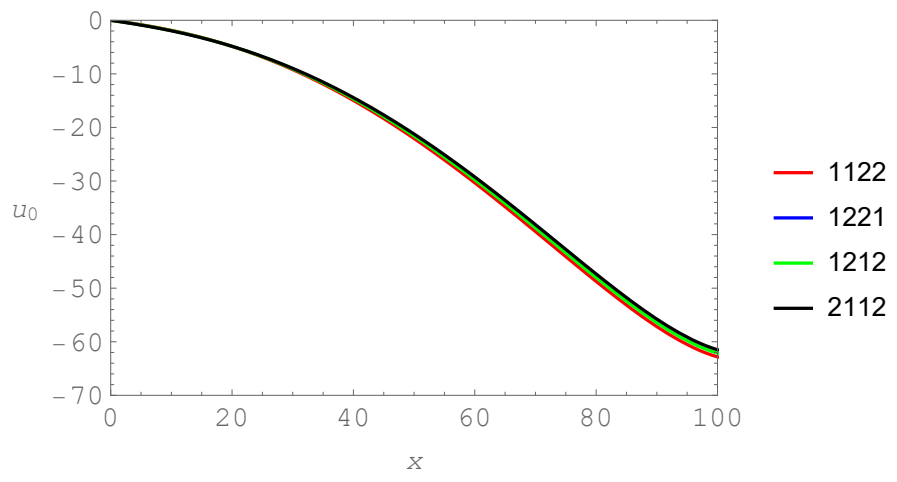

Figure 19: Axial displacements of the simply supported beam, Case (a).

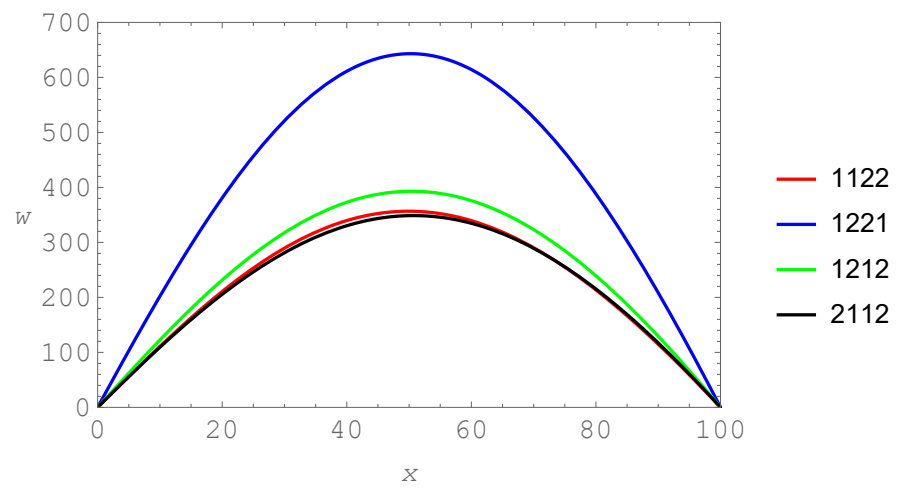

Figure 20: Transversal displacements of the simply supported beam, Case (a).

is mainly governed by the coupling effects.

In the Case (b), transverse temperature distribution also changes, so different layering schemes also affect bending behavior due to different coefficients of thermal expansions. This even causes minor loss of symmetry in transverse displacement distribution for the 1221 layer arrangement, Fig. 22. Difference is clearly visible in axial displacements, but almost identical solutions are obtained for symmetric schemes 1221 and 2112 (cannot be distinguished in Fig. 21). 


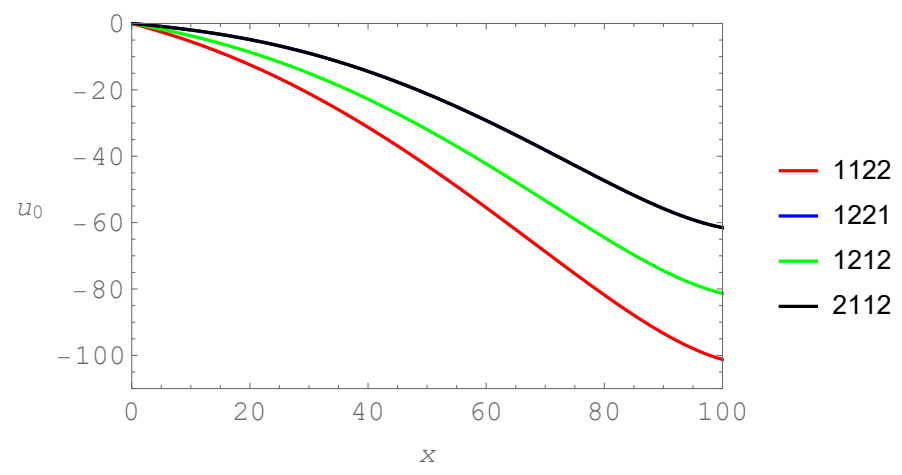

Figure 21: Axial displacements of the simply supported beam, Case (b).

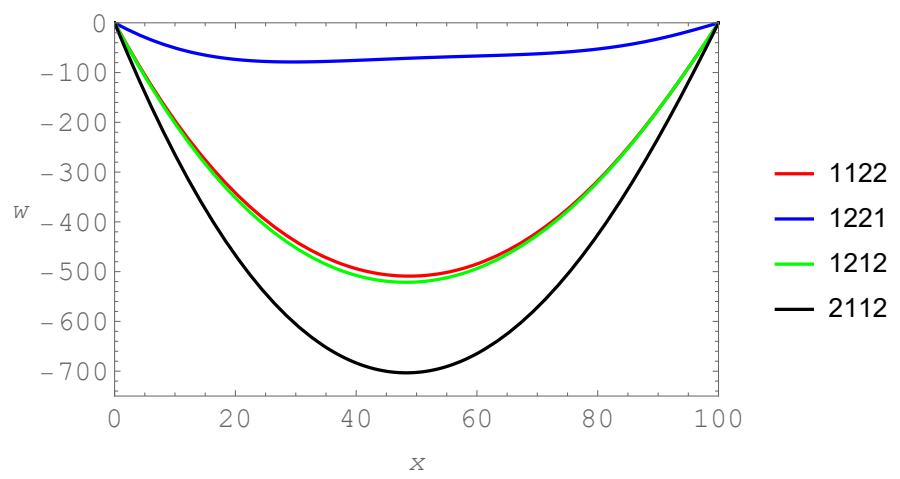

Figure 22: Transversal displacements of the simply supported beam, Case (b). 


\section{Closing remarks}

Thermomechanical analyses of multilayered micro- and nano-beams have been performed in this paper by the stress-driven nonlocal integral approach of elasticity. Main contributions and findings are summarized as follows.

- Nonisothermal extension of existing isothermal nonlocal beam stressdriven integral formulations available in literature has been provided. The conceived methodology is suitable to model and assess the nonlocal behaviour of multilayered beams assembled of arbitrary number of layers made of different rectangular cross-sections. The proposed nonlocal strategy has been shown to be able to accomodate any kind of mechanical and thermal loading.

- Presence of terms that couple axial and transverse displacements is highlighted, due to the nonlocal nature of the presented model and occur both in isothermal and nonisothermal cases. Coupling contributions can be advantageously exploited to obtain some interesting mechanical responses with potential applications in thermomechanics of nanocomposites and new-generation Micro-/Nano-Electro-MechanicalSystems (MEMS/NEMS).

- These conclusions are supported by the in-depth analysis and numerical evidence provided in section 6, where selected case-studies of applicative interest in Nanoscience and Mechanics of Composites are investigated and commented upon. The illustrated examples can also serve 
as suitable benchmarks for further developments in thermomechanics of composite nanobeams.

\section{Acknowledgments}

This work has been fully supported by Croatian Science Foundation under the project IP-2019-04-4703. This support is gratefully acknowledged.

\section{References}

[1] R. Barretta, M. Čanađija, R. Luciano, F. M. de Sciarra, Stress-driven modeling of nonlocal thermoelastic behavior of nanobeams, International Journal of Engineering Science 126 (2018) 53-67.

[2] A. Farajpour, M. H. Ghayesh, H. Farokhi, A review on the mechanics of nanostructures, International Journal of Engineering Science 133 (2018) 231-263.

[3] B. Karami, D. Shahsavari, L. Li, M. Karami, M. Janghorban, Thermal buckling of embedded sandwich piezoelectric nanoplates with functionally graded core by a nonlocal second-order shear deformation theory, Proceedings of the Institution of Mechanical Engineers, Part C: Journal of Mechanical Engineering Science 233 (1) (2019) 287-301.

[4] M. Malikan, M. Krasheninnikov, V. A. Eremeyev, Torsional stability capacity of a nano-composite shell based on a nonlocal strain gradient shell 
model under a three-dimensional magnetic field, International Journal of Engineering Science 148 (2020) 103210.

[5] L. Qi, S. Zhou, A. Li, Size-dependent bending of an electro-elastic bilayer nanobeam due to flexoelectricity and strain gradient elastic effect, Composite Structures 135 (2016) 167-175.

[6] M. A. Omari, A. Almagableh, I. Sevostianov, A. B. Yaseen, et al., Modeling of the viscoelastic properties of thermoset vinyl ester nanocomposite using artificial neural network, International Journal of Engineering Science 150 (2020) 103242.

[7] A. Eyvazian, D. Shahsavari, B. Karami, On the dynamic of graphene reinforced nanocomposite cylindrical shells subjected to a moving harmonic load, International Journal of Engineering Science 154 (2020) 103339.

[8] M. Malikan, V. A. Eremeyev, On the dynamics of a visco-piezoflexoelectric nanobeam, Symmetry 12 (4) (2020) 643.

[9] N. Tran, M. H. Ghayesh, M. Arjomandi, Ambient vibration energy harvesters: A review on nonlinear techniques for performance enhancement, International Journal of Engineering Science 127 (2018) 162-185.

[10] Z. Tadi Beni, S. Hosseini Ravandi, Y. Tadi Beni, Size-dependent nonlinear forced vibration analysis of viscoelastic/piezoelectric nano-beam, Journal of Applied and Computational Mechanics (2020). 
[11] R. Basutkar, Analytical modelling of a nanoscale series-connected bimorph piezoelectric energy harvester incorporating the flexoelectric effect, International Journal of Engineering Science 139 (2019) 42-61.

[12] S. F. Dehkordi, Y. T. Beni, Electro-mechanical free vibration of singlewalled piezoelectric/flexoelectric nano cones using consistent couple stress theory, International Journal of Mechanical Sciences 128 (2017) 125-139.

[13] T. Natsuki, K. Urakami, Analysis of vibration frequency of carbon nanotubes used as nano-force sensors considering clamped boundary condition, Electronics 8 (10) (2019) 1082.

[14] M. H. Ghayesh, H. Farokhi, Nonlinear broadband performance of energy harvesters, International Journal of Engineering Science 147 (2020) 103202.

[15] H. Vaghefpour, H. Arvin, Nonlinear free vibration analysis of preactuated isotropic piezoelectric cantilever nano-beams, Microsystem Technologies 25 (11) (2019) 4097-4110.

[16] G. Romano, R. Barretta, Stress-driven versus strain-driven nonlocal integral model for elastic nano-beams, Composites Part B: Engineering 114 (2017) 184-188.

[17] G. Romano, R. Barretta, Nonlocal elasticity in nanobeams: the stress- 
driven integral model, International Journal of Engineering Science 115 (2017) 14-27.

[18] H. M. Numanoğlu, B. Akgöz, Ö. Civalek, On dynamic analysis of nanorods, International Journal of Engineering Science 130 (2018) 3350.

[19] S. Lurie, Y. Solyaev, Revisiting bending theories of elastic gradient beams, International Journal of Engineering Science 126 (2018) 1-21.

[20] S. Lurie, Y. Solyaev, Anti-plane inclusion problem in the second gradient electroelasticity theory, International Journal of Engineering Science 144 (2019) 103129.

[21] F. P. Pinnola, M. S. Vaccaro, R. Barretta, F. M. de Sciarra, Random vibrations of stress-driven nonlocal beams with external damping, Meccanica (2020) 1-16.

[22] R. Barretta, M. Brcic, M. Canadija, R. Luciano, F. M. de Sciarra, Application of gradient elasticity to armchair carbon nanotubes: Size effects and constitutive parameters assessment, Eur. J. Mech. A. Solids 65 (2017) 1-13.

[23] M. K. Hassanzadeh-Aghdam, R. Ansari, A. Darvizeh, Micromechanical analysis of carbon nanotube-coated fiber-reinforced hybrid composites, International Journal of Engineering Science 130 (2018) 215-229. 
[24] X. Xia, G. J. Weng, D. Hou, W. Wen, Tailoring the frequencydependent electrical conductivity and dielectric permittivity of cntpolymer nanocomposites with nanosized particles, International Journal of Engineering Science 142 (2019) 1-19.

[25] V. A. Eremeyev, J.-F. Ganghoffer, V. Konopińska-Zmysłowska, N. S. Uglov, Flexoelectricity and apparent piezoelectricity of a pantographic micro-bar, International Journal of Engineering Science 149 (2020) 103213.

[26] S. Brach, L. Dormieux, D. Kondo, G. Vairo, Strength properties of nanoporous materials: A 3-layered based non-linear homogenization approach with interface effects, International Journal of Engineering Science 115 (2017) $28-42$.

[27] D. Wentzel, S. Miller, I. Sevostianov, Dependence of the electrical conductivity of graphene reinforced epoxy resin on the stress level, International Journal of Engineering Science 120 (2017) 63-70.

[28] A. Trofimov, S. Abaimov, I. Akhatov, I. Sevostianov, On the bounds of applicability of two-step homogenization technique for porous materials, International Journal of Engineering Science 123 (2018) 117-126.

[29] A. Govorov, D. Wentzel, S. Miller, A. Kanaan, I. Sevostianov, Electrical conductivity of epoxy-graphene and epoxy-carbon nanofibers composites 
subjected to compressive loading, International Journal of Engineering Science 123 (2018) 174-180.

[30] A. Trofimov, S. Abaimov, I. Sevostianov, Inverse homogenization problem: Evaluation of elastic and electrical (thermal) properties of composite constituents, International Journal of Engineering Science 129 (2018) $34-46$.

[31] H. Liu, Z. Lv, H. Wu, Nonlinear free vibration of geometrically imperfect functionally graded sandwich nanobeams based on nonlocal strain gradient theory, Composite Structures 214 (2019) 47-61.

[32] M. Canadija, R. Barretta, F. Marotti de Sciarra, On functionally graded Timoshenko nonisothermal nanobeams, Compos. Struct. 135 (2016) 286-296.

[33] E. Taati, On buckling and post-buckling behavior of functionally graded micro-beams in thermal environment, International Journal of Engineering Science 128 (2018) 63-78.

[34] A. M. Dehrouyeh-Semnani, E. Dehdashti, M. R. H. Yazdi, M. NikkhahBahrami, Nonlinear thermo-resonant behavior of fluid-conveying fg pipes, International Journal of Engineering Science 144 (2019) 103141.

[35] S. Khakalo, V. Balobanov, J. Niiranen, Modelling size-dependent bending, buckling and vibrations of $2 \mathrm{~d}$ triangular lattices by strain gradient 
elasticity models: applications to sandwich beams and auxetics, International Journal of Engineering Science 127 (2018) 33-52.

[36] M. Arefi, A. M. Zenkour, A simplified shear and normal deformations nonlocal theory for bending of functionally graded piezomagnetic sandwich nanobeams in magneto-thermo-electric environment, Journal of Sandwich Structures \& Materials 18 (5) (2016) 624-651.

[37] M. Nazemizadeh, F. Bakhtiari-Nejad, Size-dependent free vibration of nano/microbeams with piezo-layered actuators, Micro \& Nano Letters 10 (2) (2015) 93-98.

[38] F. Ebrahimi, M. Karimiasl, Nonlocal and surface effects on the buckling behavior of flexoelectric sandwich nanobeams, Mechanics of Advanced Materials and Structures 25 (11) (2018) 943-952.

[39] M. Al-shujairi, Ç. Mollamahmutoğlu, Buckling and free vibration analysis of functionally graded sandwich micro-beams resting on elastic foundation by using nonlocal strain gradient theory in conjunction with higher order shear theories under thermal effect, Composites Part B: Engineering 154 (2018) 292-312.

[40] M. Al-shujairi, Ç. Mollamahmutoğlu, Dynamic stability of sandwich functionally graded micro-beam based on the nonlocal strain gradient theory with thermal effect, Composite Structures 201 (2018) 1018-1030. 
[41] A. G. Arani, M. Pourjamshidian, M. Arefi, Influence of electro-magnetothermal environment on the wave propagation analysis of sandwich nano-beam based on nonlocal strain gradient theory and shear deformation theories, Smart Struct. Syst 20 (3) (2017) 329-342.

[42] M. Arefi, M. Pourjamshidian, A. G. Arani, Application of nonlocal strain gradient theory and various shear deformation theories to nonlinear vibration analysis of sandwich nano-beam with FG-CNTRCs face-sheets in electro-thermal environment, Applied Physics A 123 (5) (2017) 323.

[43] M. Arefi, M. Pourjamshidian, A. G. Arani, Free vibration analysis of a piezoelectric curved sandwich nano-beam with FG-CNTRCs face-sheets based on various high-order shear deformation and nonlocal elasticity theories, The European Physical Journal Plus 133 (5) (2018) 193.

[44] A. Nikpourian, M. R. Ghazavi, S. Azizi, Size-dependent secondary resonance of a piezoelectrically laminated bistable MEMS arch resonator, Composites Part B: Engineering 173 (2019) 106850.

[45] M. Sobhy, M. A. Abazid, Dynamic and instability analyses of fg graphene-reinforced sandwich deep curved nanobeams with viscoelastic core under magnetic field effect, Composites Part B: Engineering 174 (2019) 106966.

[46] O. Rahmani, S. Hosseini, H. Hayati, Frequency analysis of curved nano- 
sandwich structure based on a nonlocal model, Modern Physics Letters B 30 (10) (2016) 1650136.

[47] A. Eringen, On differential equations of nonlocal elasticity and solutions of screw dislocation and surface waves, J. Appl. Phys. 54 (9) (1983) 4703-4710.

[48] Z.-Q. Tan, Y.-C. Chen, Size-dependent electro-thermo-mechanical analysis of multilayer cantilever microactuators by Joule heating using the modified couple stress theory, Composites Part B: Engineering 161 (2019) 183-189.

[49] B. Safaei, R. Moradi-Dastjerdi, Z. Qin, F. Chu, Frequency-dependent forced vibration analysis of nanocomposite sandwich plate under thermo-mechanical loads, Composites Part B: Engineering 161 (2019) $44-54$.

[50] M. Arefi, E. Mohammad-Rezaei Bidgoli, A. M. Zenkour, Free vibration analysis of a sandwich nano-plate including FG core and piezoelectric face-sheets by considering neutral surface, Mechanics of Advanced Materials and Structures 26 (9) (2019) 741-752.

[51] A. M. Dehrouyeh-Semnani, On boundary conditions for thermally loaded FG beams, International Journal of Engineering Science 119 (2017) 109-127. 
[52] D.-G. Zhang, Y.-H. Zhou, A theoretical analysis of fgm thin plates based on physical neutral surface, Computational Materials Science 44 (2) (2008) 716-720.

[53] T. Morimoto, Y. Tanigawa, R. Kawamura, Thermal buckling of functionally graded rectangular plates subjected to partial heating, Int. J. Mech. Sci. 48 (9) (2006) 926-937.

[54] S. Abrate, Functionally graded plates behave like homogeneous plates, Composites Part B: Engineering 39 (1) (2008) 151-158.

[55] M. Rezaiee-Pajand, M. Mokhtari, Size dependent buckling analysis of nano sandwich beams by two schemes, Mechanics of Advanced Materials and Structures 27 (12) (2020) 975-990.

[56] H. Wu, H. Liu, Nonlinear thermo-mechanical response of temperaturedependent FG sandwich nanobeams with geometric imperfection, Engineering with Computers (2020) 1-21.

[57] N. Kammoun, H. Jrad, S. Bouaziz, M. Amar, M. Soula, M. Haddar, Thermo-electro-mechanical vibration characteristics of graphene/piezoelectric/graphene sandwich nanobeams, Journal of Mechanics 35 (1) (2019) 65-79.

[58] N. Kammoun, H. Jrad, S. Bouaziz, M. Soula, M. Haddar, Vibration analysis of three-layered nanobeams based on nonlocal elasticity theory, Journal of Theoretical and Applied Mechanics 55 (2017). 
[59] O. Rahmani, S. Ghaffari, Frequency analysis of nano sandwich structure with nonlocal effect, in: Advanced Materials Research, Vol. 829, Trans Tech Publ, 2014, pp. 231-235.

[60] R. Barretta, M. Čanađija, F. M. d. Sciarra, Nonlocal mechanical behavior of layered nanobeams, Symmetry 12 (5) (2020) 717.

[61] G. Romano, R. Barretta, M. Diaco, F. Marotti de Sciarra, Constitutive boundary conditions and paradoxes in nonlocal elastic nanobeams, Int. J. Mech. Sci. 121 (2017) 151-156.

[62] A. Apuzzo, R. Barretta, S. Faghidian, R. Luciano, F. M. de Sciarra, Nonlocal strain gradient exact solutions for functionally graded inflected nano-beams, Composites Part B: Engineering 164 (2019) 667 - 674.

[63] R. Barretta, M. Čanadija, L. Feo, R. Luciano, F. M. de Sciarra, R. Penna, Exact solutions of inflected functionally graded nano-beams in integral elasticity, Composites Part B: Engineering 142 (2018) 273 286.

[64] R. Barretta, M. Čanađija, F. M. de Sciarra, Nonlocal integral thermoelasticity: A thermodynamic framework for functionally graded beams, Composite Structures 225 (2019) 111104. doi:10.1016/j . compstruct. 2019.111104.

[65] L. O. Larbi, A. Kaci, M. S. A. Houari, A. Tounsi, An efficient shear deformation beam theory based on neutral surface position for bend- 
ing and free vibration of functionally graded beams, Mech. Based Des. Struct. Mach. 41 (4) (2013) 421-433.

[66] A. D. Polyanin, A. V. Manzhirov, Handbook of integral equations, CRC, Boca Raton, FL (1998).

[67] N. Challamel, C. Wang, The small length scale effect for a non-local cantilever beam: a paradox solved, Nanotechnology 19 (34) (2008) 345703.

[68] J. Peddieson, G. R. Buchanan, R. P. McNitt, Application of nonlocal continuum models to nanotechnology, International Journal of Engineering Science 41 (3-5) (2003) 305-312.

[69] R. Hetnarski, M. Eslami, Thermal Stresses-Advanced Theory and Applications, Springer, New York, 2009.

[70] N. Noda, R. Hetnarski, Y. Tanigawa, Thermal Stresses, Taylor \& Francis, 2003.

[71] B. A. Boley, J. H. Weiner, Theory of thermal stresses, John Wiley

[72] S. Corkovic, R. W. Whatmore, Q. Zhang, Development of residual stress in sol-gel derived $\mathrm{Pb}(\mathrm{Zr}, \mathrm{Ti}) \mathrm{O}_{3}$ films: An experimental study, Journal of Applied Physics 103 (8) (2008) 084101. 